\title{
Quantitative phase-field modeling for boiling phenomena
}

\author{
Arnoldo Badillo* \\ Thermal-Hydraulics Laboratory, Paul Scherrer Institute, CH-5232 Villigen PSI, Switzerland
}

(Received 6 August 2012; published 16 October 2012)

\begin{abstract}
A phase-field model is developed for quantitative simulation of bubble growth in the diffusion-controlled regime. The model accounts for phase change and surface tension effects at the liquid-vapor interface of pure substances with large property contrast. The derivation of the model follows a two-fluid approach, where the diffuse interface is assumed to have an internal microstructure, defined by a sharp interface. Despite the fact that phases within the diffuse interface are considered to have their own velocities and pressures, an averaging procedure at the atomic scale, allows for expressing all the constitutive equations in terms of mixture quantities. From the averaging procedure and asymptotic analysis of the model, nonconventional terms appear in the energy and phase-field equations to compensate for the variation of the properties across the diffuse interface. Without these new terms, no convergence towards the sharp-interface model can be attained. The asymptotic analysis also revealed a very small thermal capillary length for real fluids, such as water, that makes impossible for conventional phase-field models to capture bubble growth in the millimeter range size. For instance, important phenomena such as bubble growth and detachment from a hot surface could not be simulated due to the large number of grids points required to resolve all the scales. Since the shape of the liquid-vapor interface is primarily controlled by the effects of an isotropic surface energy (surface tension), a solution involving the elimination of the curvature from the phase-field equation is devised. The elimination of the curvature from the phase-field equation changes the length scale dominating the phase change from the thermal capillary length to the thickness of the thermal boundary layer, which is several orders of magnitude larger. A detailed analysis of the phase-field equation revealed that a split of this equation into two independent parts is possible for system sizes ranging from micrometers and above. The split of the phase-field equation leads to two equations controlling independently the phase change and the shape of the phase-field profile across the diffuse interface. A new algorithm is presented to solve a fully coupled model, including advection, phase change, and surface tension effects. Good agreement is found among numerical simulations, analytical solutions, and experimental results.
\end{abstract}

DOI: 10.1103/PhysRevE.86.041603

PACS number(s): 68.03.-g, 64.70.fh, 47.55.N-

\section{INTRODUCTION}

Boiling is a common phenomenon found in many scientific and industrial applications, where high heat transfer coefficients between a hot surface and a cooling fluid can be achieved. This efficient heat transfer mechanism is what allows us to have air-conditioning systems and refrigerators in our homes, but it also can lead to dangerous situations in boiling water nuclear reactors in the eventuality of reaching the critical heat flux [1]. Improving our understanding on boiling would not only increase the safety of the nuclear industry, but it also could help us to design, for instance, more efficient cooling system for microprocessors [2]. Broadly speaking, boiling occurs when a liquid is heated above its saturation temperature, generating the appropriate conditions for nucleation and growth of vapor bubbles. Many questions arise when we think in the fundamental physical phenomena governing this complex phase change; for example, What is the influence of a force field (like gravity or magnetic fields), What are the length and times scales relevant to the dynamic of the interface, What is the relationship between superheating and interface velocity or What are the effects of the surface properties? Some of these questions are still unanswered and probably will require new experimental tools to be clarified. A good example is the poorly understood evaporation or condensation phenomena at the contact line,

*arnoldo.badillo@psi.ch where solid, liquid, and vapor meet. Some authors $[3,4]$ state that during boiling, most of the vapor is generated in a tiny region around the contact line, where high heat fluxes are expected. Others [5], and based on their own experimental observations, disagree with this idea and claim that evaporation at the contact line accounts only for a fraction of the total evaporation rate. Whatever the reality at the contact line is, it is undeniable that to perform accurate simulations of boiling, we must first develop appropriate models to describe the phase change at the liquid-vapor interface and then incorporate the wall effects at the contact line.

The study of boiling, from a theoretical point of view, started at the beginning of the 20th century, arguably with the work of Lord Rayleigh [6], who developed a simple analytical solution for vapor bubbles growing in the inertia-controlled regime. This regime is found in the early stages of bubble growth, where the interface temperature is higher than the saturation value. Under these conditions, the interface is superheated and, consequently, does not require a net heat flux to drive the evaporation process. In this regime, the bubble radius $R$ increases linearly with time $R(t) \sim t$, whereas in the diffusion-controlled regime, bubbles grow proportionally to the square root of time $R(t) \sim t^{\frac{1}{2}}$. These two regimes represent the limiting cases, where the net evaporation rate in the former is controlled by kinetic effects and by thermal diffusion in the latter. This particular fact shows us the need for developing a model that can, in principle, predict the phase change in both regimes. For bubbles growing and detaching from a heating 
surface, kinetic effects might play an important role in the overall process of boiling, which have never been accounted for.

Most of the models developed until now focus on the diffusion-controlled regime, where the thickness of the thermal boundary layer sets the length scale dominating the evaporation process. Different formulations based on sharp-interface [7-11] or diffuse-interface models [4] have been used to predict the velocity of the interface due to phase change. In these formulations, the evaporation rate is obtained from the Stefan condition, which is basically a restatement of the conservation of energy at a sharp interface under local thermodynamic equilibrium (the interface temperature is at the saturation value in the absence of curvature). This condition basically states that the mass evaporation rate per unit surface $\dot{\Gamma}$ can be related to the latent heat $L$ and the net heat flux to the interface by $L \dot{\Gamma}=\left(\mathbf{q}_{l}-\mathbf{q}_{v}\right) \cdot \mathbf{n}$, where $\mathbf{n}$ is the normal vector and the subindexes $l$ and $v$ represent the heat fluxes at the liquid and vapor side of the interface, respectively. Models using the Stefan condition to calculate the evaporation rate can be very accurate when the thermal boundary layer is well resolved, but simplicity comes at a price. On the one hand, these models cannot include kinetic or curvature effects; on the other hand, numerical implementation is not straightforward. Although new models, such as the one developed by Sato and Ničeno [8], alleviate the burden of numerical implementation, they are still bound to the diffusion-controlled regime, missing the potential important contribution from kinetic and curvature effects. An alternative to these models is, for instance, the temperature recovery method [12], where the evaporation rate is obtained from the local superheating. In this model, whenever a computational cell exceeds the saturation temperature, phase change takes place at a rate given by $L \dot{\Gamma}=\rho c_{P} \Delta T$, where $\Delta T$ is the local superheating. Nonetheless, the model requires the liquid to be at the saturation temperature, leaving out the possibility of simulating superheated fluids. In addition, the time scale embedded in the evaporation rate is completely arbitrary. In order to develop a general model for boiling phenomena, a phase-field approach is adopted, which provides a robust framework to derive the constitutive equations from very basic principles.

For the past two decades, the development of phase-field models has been characterized by their success on simulating complex morphological changes during phase transitions. For instance, quantitative models for dendritic solidification have opened a new window for a deeper understanding of the fundamental mechanisms controlling the solidification of metallic alloys [13-16]. Due to their powerful and yet simple basic ideas, phase-field models have drawn the attention of scientists working in several fields such as dislocation dynamics [17], grain growth [18], and quantum dot formation [19] just to name a few. In the field of multiphase flows, we can also find several variants of phase-field formulations to study phase separation of binary fluids under shear stress [20], microfluidics [21], bubble and drop dynamics [22], and even an interface tracking technique based on the phase-field equation [23]. The literature on phase-field modeling of two phase flows is extensive, but, to the best of my knowledge, a quantitative model including phase change has not been presented yet. Borcia and Bestehorn [24] derived a phase-field model to study the influence of phase change on Marangoni convection, but they did not provide an expression for the evaporation rate in terms of the local superheating, nor an exhaustive validation against known analytical solutions. Jamet et al. [25] derived a phase-field model, which he and coworkers called the "second gradient method' for liquid-vapor flows with phase change, but the authors did not present a rigorous asymptotic analysis of the model. In addition, the time scale controlling the interface velocity due to phase change was chosen arbitrarily in the solution of the Stefan problem.

Although quantitative phase-field models for dendritic solidification of pure substances can be traced back to the pioneer work of Karma and Rappel [26] in 1998, the extension of this model to evaporating systems is not straightforward. The main reason for this is the large density ratio between the two phases in coexistence, which induces a mass flow in the direction normal to the interface during evaporation or condensation. This flow, aside from transporting mass, also carries energy with it. The correct formulation of the energy and phase-field equations, which is presented in this work, turned out to be vital for the convergence of the model towards the sharp-interface solution in the diffusion-controlled regime.

The present article is organized as follows. In Sec. II, the constitutive equations of the phase-field model are derived from a single fluid and a two-phase flow formulations. A thermodynamic derivation of the surface tension force is presented in Sec. III. The derivation of the evaporation rate is given in Sec. IV. The asymptotic analysis of the model is presented in Sec. V and the elimination of the curvature from the phase-field model is presented in Sec. VI. The algorithm to solve the model is described in Sec. VII. Section VIII presents the comparison of phase-field simulations with analytical solutions and experimental results. Conclusions are drawn in Sec. IX. Appendix A presents the basic idea behind the derivation of the phase-field equation for conserved and nonconserved order parameters. Appendix B shows the derivation of the differential operators used in the asymptotic analysis for a three-dimensional curvilinear orthogonal coordinate system. Appendix C includes a set of integrals relevant for the derivation of the model.

\section{DERIVATION OF THE CONSTITUTIVE EQUATIONS}

The constitutive field equations can be derived essentially in two ways: (1) considering a single fluid with sharply (but smoothly) varying properties across the transition region [24] and (2) assuming an internal microstructure within the liquid-vapor interface, where two bulk phases coexist and are separated by a sharp interface at the atomic scale [27].

\section{A. Single-fluid formulation}

Although the derivation of the constitutive equations based on a single fluid is simple and straightforward, it has a fundamental flaw that impeded the convergence of the model towards the sharp-interface limit. Nonetheless, its derivation is important to illustrate why this approach is not suitable for evaporating systems. Neglecting viscous dissipation, the mass, momentum, and energy equations can be expressed in 
their nonconservative form as

$$
\begin{aligned}
\nabla \cdot \mathbf{u} & =-\frac{1}{\rho} \frac{D \rho}{D t}, \\
\rho \frac{D \mathbf{u}}{D t} & =-\nabla \cdot \stackrel{\leftrightarrow}{\boldsymbol{\sigma}}+\mathbf{f}, \\
\rho \frac{D h}{D t} & =-\nabla \cdot \mathbf{q}+S,
\end{aligned}
$$

where $D$ is the material derivative, $\stackrel{\leftrightarrow}{\sigma}$ the stress tensor (including viscous and surface tension effects), $\mathbf{f}$ an external force, $h$ the enthalpy per unit mass, $\mathbf{q}$ the heat flux, and $S$ a source term. For a quasi-incompressible formulation, the density does not depend on pressure, but it can vary with other thermodynamic variables such as the temperature, phase-field, concentration, and so on. If the density only depends on the phase field, Eq. (1a) can be expressed as

$$
\nabla \cdot \mathbf{u}=-\frac{1}{\rho} \frac{\partial \rho}{\partial \phi} \frac{D \phi}{D t} .
$$

To introduce the mass evaporation rate, we must assume a certain variation of the density with the phase field. Molecular dynamic simulations of saturated water in contact with its vapor [28] have shown that the density profile across the diffuse interface varies in a hyperbolic tangent fashion, this is

$$
\begin{aligned}
\rho(\phi) & =\rho_{l} \phi+\rho_{v}(1-\phi), \\
\phi & =[1+\tanh (x / W \sqrt{2})] / 2,
\end{aligned}
$$

where $\rho_{l}$ and $\rho_{v}$ are bulk densities and $W$ is a measure of the interface thickness. Making connection to sharp-interface formulations, we introduce the evaporation rate as

$$
\boldsymbol{\nabla} \cdot \mathbf{u}=-\left(\frac{1}{\rho_{l}}-\frac{1}{\rho_{v}}\right) \ddot{\Gamma} .
$$

It is noteworthy that since no expression has been provided for the evaporation rate $\ddot{\Gamma}$, Eq. (4) represents an alternative for writing Eq. (2), where no assumptions have been made. From Eqs. (2) and (4), the evolution of the phase field reads

$$
\frac{D \phi}{D t}=-\frac{\rho}{\rho_{l} \rho_{v}} \ddot{\Gamma},
$$

and, in its conservative form,

$$
\frac{\partial \phi}{\partial t}+\nabla \cdot(\mathbf{u} \phi)=-\frac{\ddot{\Gamma}}{\rho_{l}} .
$$

To arrive to the final form of the energy equation, it is assumed that the enthalpy is only a function of the temperature and phase field. Hence, its material derivative is given by

$$
\frac{D h}{D t}=\frac{\partial h}{\partial \phi} \frac{D \phi}{D t}+\frac{\partial h}{\partial T} \frac{D T}{D t} .
$$

Since $h$ is a thermodynamic variable per unit mass, an appropriate interpolation function for the enthalpy should consider the mass fraction instead of the phase field itself, this is $h=h\left(\chi_{l}, \chi_{v}\right)$, where the mass fraction is defined as $\chi_{i}=\rho_{i} \phi_{i} / \rho$. By choosing a linear interpolation based on the mass fraction $h=h_{l} \chi_{l}+h_{v} \chi_{v}$ and replacing Eq. (5) into
Eq. (7) we arrive at

$$
\frac{D h}{D t}=\left(c_{P}^{l} \chi_{l}+c_{P}^{v} \chi_{v}\right) \frac{D T}{D t}+\frac{L}{\rho} \ddot{\Gamma},
$$

where the thermodynamic definition for the heat capacity at constant pressure $c_{P}^{i}=\left(\partial h_{i} / \partial T\right)_{P}$ and latent heat per unit mass $L=h_{l}-h_{v}$ have been used. Defining the variation of the heat capacity across the diffuse interface as $c_{P}=c_{P}^{l} \chi_{l}+$ $c_{P}^{v} \chi_{v}$ and replacing Eq. (8) into Eq. (1c) leads us to the final expression of the energy equation,

$$
\rho c_{P} \frac{D T}{D t}=-\nabla \cdot \mathbf{q}-L \ddot{\Gamma}+S .
$$

This allows us to write an equivalent form of the Eqs. (1a)-(1c) in terms of the evaporation rate as

$$
\begin{aligned}
\nabla \cdot \mathbf{u} & =-\left(\frac{1}{\rho_{l}}-\frac{1}{\rho_{v}}\right) \ddot{\Gamma}, \\
\frac{\partial \phi}{\partial t}+\nabla \cdot(\mathbf{u} \phi) & =-\frac{\ddot{\Gamma}}{\rho_{l}}, \\
\rho \frac{D \mathbf{u}}{D t} & =-\nabla \cdot \stackrel{\leftrightarrow}{\sigma}+\mathbf{f}, \\
\rho c_{P} \frac{D T}{D t} & =-\nabla \cdot \mathbf{q}-L \ddot{\Gamma}+S .
\end{aligned}
$$

Be aware that to satisfy mass conservation Eq. (1a), we have to satisfy simultaneously Eqs. (10a) and (10b). This set of equations, although very familiar and appealing for dealing with boiling, has a fundamental problem in the advection of the phase field and sensible heat. Traditionally, the terms $\mathbf{u} \phi$ and $\rho c_{P} \mathbf{u} \cdot \nabla T$ are linear on the phase field and temperature, respectively (for constant bulk properties), but in the presence of phase change with density difference, the velocity field varies sharply across the diffuse interface with the phase field $\mathbf{u} \equiv \mathbf{u}(\phi)$ and nonlinear terms arise. These new nonlinear terms impede the convergence of this model towards the sharp-interface solution in the framework of conventional phase-field modeling. Although an asymptotic analysis of these equations could shine some light on how to eliminate the problems caused by the nonlinear terms, all efforts spent in this task did not produce any fruitful results. To further clarify the effects of nonlinear terms, we can analyze the one-dimensional problem presented schematically in Fig. 1. In this case, the vapor is enclosed by a wall and the liquid phase, thus, as the phase change takes place, a certain amount of liquid must exit the system at the right boundary to compensate for the expansion of the liquid during the evaporation process. From a sharp-interface point of view, a mass balance at the interface requires $\rho_{l}\left(\mathbf{u}_{l}-\mathbf{u}_{i}\right) \cdot \mathbf{n}=\rho_{v}\left(\mathbf{u}_{v}-\mathbf{u}_{i}\right) \cdot \mathbf{n}$, therefore,

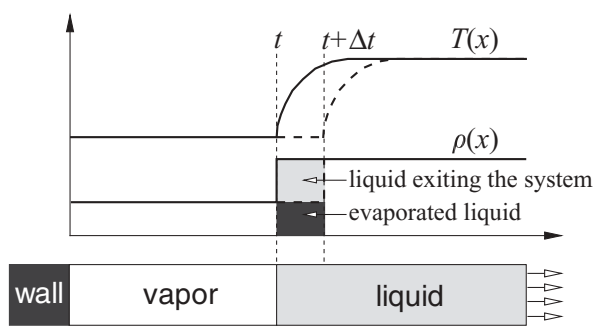

FIG. 1. One-dimensional evaporating system. 
if the vapor is stationary $\left(\mathbf{u}_{v}=0\right)$, the velocity of the liquid is related to the interface velocity $\mathbf{u}_{i}$ through $\mathbf{u}_{l}=\mathbf{u}_{i}\left(\rho_{l}-\right.$ $\left.\rho_{v}\right) / \rho_{l}$. For this particular case, the evaporation rate per unit volume can be directly related to the interface velocity by $\ddot{\Gamma}=\rho_{v} \mathbf{u}_{i} \cdot \mathbf{n} \delta\left(x-x_{i}\right)$, where $\delta\left(x-x_{i}\right)$ is the $\delta$ function, $x_{i}$ the interface position, and $\mathbf{n}$ the normal vector pointing towards the liquid phase. The diffuse interface version of this expression for a phase field propagating with an invariant profile is given by $\ddot{\Gamma}=\rho_{v} \mathbf{u}_{i} \cdot \nabla \phi$. Replacing this expression into the mass conservation equation Eq. (10a) and integrating, leads to the following velocity profile across the interface

$$
u(\phi)=\left(\frac{\rho_{l}-\rho_{v}}{\rho_{l}}\right) u_{i} \phi .
$$

The velocity in the liquid phase $(\phi=1)$ clearly reduces to the same value found from the sharp-interface solution. Replacing Eq. (11) into Eq. (10b) yields

$$
\frac{\partial \phi}{\partial t}=-u_{i} \frac{\partial \phi}{\partial x}\left[r_{v l}+\left(1-r_{v l}\right) 2 \phi\right],
$$

where $r_{v l}=\rho_{v} / \rho_{l}$ is the density ratio. The term inside the parenthesis in Eq. (12) induces a deformation of the phase-field profile, which prevents its propagation with the conventional hyperbolic tangent profile given in Eq. (3b). For the case of equal densities, Eq. (12) reduces to $\partial_{t} \phi=-u_{i} \partial_{x} \phi$. Despite that the hyperbolic tangent profile cannot be a solution of Eq. (12) for a density ratio different than unity, the integral of the right-hand side of Eq. (12) will always reduce to $-u_{i}$ if $\partial_{x} \phi$ is an even function, independently of the value of the density ratio. This simple observation provides a path to eliminate the spurious effects of nonlinear terms in the phase-field equation, which will be discussed in subsequent sections. The problem with the advection of sensible heat will be better understood after the derivation of the energy equation from a two-phase flow formulation.

\section{B. Two-phase flow averaged model}

The second approach provides us with a cleaner derivation, where the interpolation functions arise naturally from the averaging process. Sun and Beckermann [27] derived a phasefield model using a two-phase flow approach, but in their paper no explicit treatment of the phase change was provided. In the present derivation, I have followed a similar route but with a different physical interpretation of the physical phenomena occurring inside the diffuse interface. The fundamentals for the averaging technique have been presented extensively elsewhere [29] and, therefore, I will provide only the most relevant identities and results. The main idea in a two-phase flow approach is the existence of a mathematical surface (sharp interface), which mediates the transfer of several quantities between two phases in contact. In contrast to the derivation of Sun and Beckermann [27], in the present work, no attempt was made to relate the hypothetical surface to possible atomic structures within the diffuse interface. Hence this surface shall be understood only as a mathematical tool to simplify the derivation of the model. The main reason for this is that inside the real transition region (whose thickness is usually only few atomic radii), atoms and/or molecules differ in their behavior from that in the bulk and, therefore, they cannot be associated

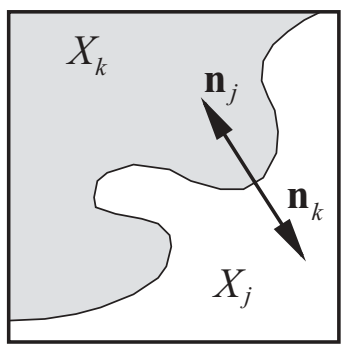

FIG. 2. Control volume used in the averaging procedure.

to any of the two phases. Furthermore, short- and long-range atomic interactions are in evident contrast to the idea of an atomic structure, represented by a sharp interface, within the diffuse-interface region. A schematic of a control volume used for the derivation is presented in Fig. 2. To represent each phase, we define a function $X_{k}(\mathbf{x})$ that depends on position and is equal to 1 in phase $k$ and 0 otherwise. From this elemental definition, all the identities found in the averaging process can be easily derived. In our case, we will, first, perform a volumetric average to derive the equations and then use an ensemble average to recover the macroscopic behavior of the interface. Thus, the volumetric average of any quantity within its own volume $V_{k}$ and within the control volume $V$ is defined as

$$
\begin{aligned}
\left\langle\Phi_{k}\right\rangle^{k} & =\frac{1}{V_{k}} \int_{V_{k}}\left(\Phi_{k} X_{k}\right) d V_{k}, \\
\left\langle\Phi_{k}\right\rangle & =\frac{1}{V} \int_{V}\left(\Phi_{k} X_{k}\right) d V .
\end{aligned}
$$

The volume fraction of phase $k$ is, thus, given by $\phi_{k}=\left\langle X_{k}\right\rangle$. Defining the normal vector of phase $k$ as $\mathbf{n}_{k}=-\nabla X_{k} /\left|\nabla X_{k}\right|$, we derive at once

$$
\left\langle\frac{\partial \Phi_{k}}{\partial t}\right\rangle=\frac{\partial\left\langle\Phi_{k}\right\rangle}{\partial t}-\frac{1}{V} \int_{A_{k}} \Phi_{k} \mathbf{u}_{i} \cdot \mathbf{n}_{k} d A_{k} .
$$

A deviation (or fluctuation) of $\Phi_{k}$ from its volumetric average is defined as

$$
\hat{\Phi}_{k}=\Phi_{k}-\left\langle\Phi_{k}\right\rangle^{k}
$$

from which we derive

$$
\left\langle\Psi_{k} \Phi_{k}\right\rangle^{k}=\left\langle\Psi_{k}\right\rangle^{k}\left\langle\Phi_{k}\right\rangle^{k}+\left\langle\hat{\Psi}_{k} \hat{\Phi}_{k}\right\rangle^{k}
$$

These definitions set the mathematical framework for the current derivation.

\section{Averaged mass conservation equation}

Defining the linear momentum in phase $k$ as $\mathbf{p}_{k}=\rho_{k} \mathbf{u}_{k}$, the averaged mass conservation equation for phase $k$ is given by

$$
\left\langle\frac{\partial \rho_{k}}{\partial t}+\nabla \cdot \mathbf{p}_{k}\right\rangle=0
$$

which, after some algebra, can be expressed as

$$
\frac{\partial\left(\phi_{k}\left\langle\rho_{k}\right\rangle^{k}\right)}{\partial t}+\nabla \cdot\left(\phi_{k}\left\langle\mathbf{u}_{k}\right\rangle^{k}\left\langle\rho_{k}\right\rangle^{k}\right)=-\ddot{\Gamma}_{k}-\nabla \cdot\left(\phi_{k}\left\langle\hat{\mathbf{u}}_{k} \hat{\rho}_{k}\right\rangle^{k}\right),
$$


where the interfacial mass transfer rate per unit volume from phase $k$ to a neighboring phase is defined as

$$
\ddot{\Gamma}_{k}=\frac{1}{V} \int_{A_{k}} \rho_{k}\left(\mathbf{u}_{k}-\mathbf{u}_{i}\right) \cdot \mathbf{n}_{k} d A_{k} .
$$

By defining a mixture density as $\bar{\rho}=\left\langle\rho_{l}\right\rangle^{l} \phi_{l}+\left\langle\rho_{v}\right\rangle^{v} \phi_{v}$ and adding the equations for the liquid and vapor, we get

$$
\begin{gathered}
\frac{\partial \bar{\rho}}{\partial t}+\nabla \cdot\left(\phi_{l}\left\langle\mathbf{u}_{l}\right\rangle^{l}\left\langle\rho_{l}\right\rangle^{l}+\phi_{v}\left\langle\mathbf{u}_{v}\right\rangle^{v}\left\langle\rho_{v}\right\rangle^{v}\right) \\
=-\nabla \cdot\left(\phi_{l}\left\langle\hat{\mathbf{u}}_{l} \hat{\rho}_{l}\right\rangle^{l}+\phi_{v}\left\langle\hat{\mathbf{u}}_{v} \hat{\rho}_{v}\right\rangle^{v}\right) .
\end{gathered}
$$

Although the definition for the mixture density is identical to that in Eq. (3a), there is a subtle difference in their physical meaning. In the single-fluid formulation, the density is understood as a property of a fluid that varies rapidly across the interface, whereas in the two-phase flow formulation, the phase field represents a volume fraction and the density is seen as a volume-averaged quantity between two coexisting phases. To arrive to Eq. (21), the condition for mass conservation at a sharp interface $\rho_{l}\left(\mathbf{u}_{l}-\mathbf{u}_{i}\right) \cdot \mathbf{n}_{l}+\rho_{v}\left(\mathbf{u}_{v}-\mathbf{u}_{i}\right) \cdot \mathbf{n}_{v}=0$ was implicitly used. Equation (21) is identical to that derived by Sun and Beckermann [27] in the case of constant bulk densities. The same authors used a mass-weighted average to define a mixture velocity as $\overline{\mathbf{u}}^{*}=\left(\phi_{l}\left\langle\mathbf{u}_{l}\right\rangle^{l}\left\langle\rho_{l}\right\rangle^{l}+\phi_{v}\left\langle\mathbf{u}_{v}\right\rangle^{v}\left\langle\rho_{v}\right\rangle^{v}\right) / \bar{\rho}$. For a constant bulk density, Eq. (19) can be written as

$$
\frac{\partial \phi_{k}}{\partial t}+\nabla \cdot\left(\phi_{k}\left\langle\mathbf{u}_{k}\right\rangle^{k}\right)=-\frac{\ddot{\Gamma}_{k}}{\rho_{k}} .
$$

Adding Eq. (22) for both phases and applying volume conservation $\phi_{l}+\phi_{v}=1$ leads to

$$
\nabla \cdot \overline{\mathbf{u}}=-\left(\frac{1}{\rho_{l}}-\frac{1}{\rho_{v}}\right) \ddot{\Gamma}_{l},
$$

where a new mixture velocity, based on a volume-weighted average, has been defined

$$
\overline{\mathbf{u}}=\phi_{l}\left\langle\mathbf{u}_{l}\right\rangle^{l}+\phi_{v}\left\langle\mathbf{u}_{v}\right\rangle^{v} .
$$

Since the velocity of the fluid is an intrinsic volumetric quantity, it seems more logical to define a mixture velocity based on a volume-weighted average rather than a massweighted average as Sun and Beckermann did. Although Eq. (23) looks identical to Eq. (17) derived by these authors [27], an important difference arises from the definition of the mixture velocity. In their work, Eq. (17) is only valid under the assumption of equal velocities in both phases; consequently, additional terms (easily derived from their equations) must be included to account properly for the expansion or contraction of one of the two phases, in the presence of phase change with density difference. Since the assumption of equal velocities was not made in the present derivation, Eq. (23) is complete and holds in all situations, even in the presence of phase change with density difference. It is important to remark that in the absence of slip between the two phases (which is the case for pure substances), equal velocities can only be achieved in the absence of phase change or by taking equal densities. This can be easily proved by applying mass conservation at the interface $\ddot{\Gamma}_{l}+\ddot{\Gamma}_{v}=0$, this is

$$
\int_{A_{l}}\left(\mathbf{u}_{l}-\mathbf{u}_{v}\right) \cdot \mathbf{n}_{l} d A_{l}=\left(\frac{1}{\rho_{l}}-\frac{1}{\rho_{v}}\right) \ddot{\Gamma}_{l} .
$$

Equation (25) shows that assuming equal velocities $\left(\mathbf{u}_{l}-\mathbf{u}_{v}=\right.$ 0 ) within the diffuse interface is indeed incorrect in the presence of phase change with density difference.

\section{Averaged momentum equation}

The averaged momentum equation for phase $k$ reads

$$
\left\langle\frac{\partial \mathbf{p}_{k}}{\partial t}+\nabla \cdot\left(\mathbf{u}_{k} \mathbf{p}_{k}\right)\right\rangle=\left\langle-\nabla \cdot \overleftrightarrow{\sigma_{k}}+\mathbf{f}_{k}\right\rangle .
$$

Applying the same averaging procedure leads to

$$
\begin{aligned}
& \frac{\partial\left\langle\mathbf{p}_{k}\right\rangle}{\partial t}+\nabla \cdot\left\langle\mathbf{u}_{k} \mathbf{p}_{k}\right\rangle \\
& =-\nabla \cdot\left\langle\overrightarrow{\boldsymbol{\sigma}_{k}}\right\rangle-\frac{1}{V} \int_{A_{k}}\left[\overleftrightarrow{\boldsymbol{\sigma}_{k}}+\mathbf{p}_{k}\left(\mathbf{u}_{k}-\mathbf{u}_{i}\right)\right] \cdot \mathbf{n}_{k} d A_{k}+\left\langle\mathbf{f}_{k}\right\rangle .
\end{aligned}
$$

After lengthy algebra and changing the nomenclature for the averaged quantities in phase $k$ as $\left\langle\Phi_{k}\right\rangle^{k}=\bar{\Phi}_{k}$ we arrive to

$$
\begin{aligned}
& \frac{\partial\left(\phi_{k} \overline{\mathbf{u}}_{k} \bar{\rho}_{k}\right)}{\partial t}+\nabla \cdot\left(\phi_{k} \overline{\mathbf{u}}_{k} \overline{\mathbf{u}}_{k} \bar{\rho}_{k}\right) \\
& =-\nabla \cdot\left(\left\langle\overleftrightarrow{\boldsymbol{\sigma}_{k}}\right\rangle+\phi_{k} \bar{\rho}_{k}\left\langle\hat{\mathbf{u}}_{k} \hat{\mathbf{u}}_{k}\right\rangle^{k}\right)+\phi_{k} \overline{\mathbf{f}}_{k}+\mathbf{f}_{k, i}+\mathbf{f}_{k}^{\hat{\rho}},
\end{aligned}
$$

where $\mathbf{f}_{k, i}=-\frac{1}{V} \int_{A_{k}}\left[\overleftrightarrow{\boldsymbol{\sigma}_{k}}+\mathbf{p}_{k}\left(\mathbf{u}_{k}-\mathbf{u}_{i}\right)\right] \cdot \mathbf{n}_{k} d A_{k}$ is the total force exerted from phase $k$ to the neighbor phase and $\mathbf{f}_{k}^{\hat{\rho}}=\partial_{t}\left(\phi_{k}\left\langle\hat{\mathbf{u}}_{k} \hat{\rho}_{k}\right\rangle^{k}\right)+\nabla \cdot\left(\phi_{k}\left\langle\hat{\rho}_{k} \hat{\mathbf{u}}_{k} \hat{\mathbf{u}}_{k}\right\rangle^{k}\right)$ is a fluctuating force arising from density and velocity fluctuations. As in the framework of large eddy simulations (LES) in turbulent flows modeling, the term $\stackrel{\leftrightarrow}{\boldsymbol{\tau}}_{R e}^{(k)}=\bar{\rho}_{k}\left\langle\hat{\mathbf{u}}_{k} \hat{\mathbf{u}}_{k}\right\rangle^{k}$ also requires subgrid correlation functions. In Reynolds-averaged Navier-Stokes equations (RANS), a similar term appears when averaging in time, leading to the well-known Reynolds stress tensor. In resemblance to those models, I will make use of the subindex $\mathrm{Re}$ to refer to this tensor. Adding Eq. (28) for both phases yields

$$
\begin{gathered}
\frac{\partial\left(\phi_{l} \overline{\mathbf{u}}_{l} \bar{\rho}_{l}+\phi_{v} \overline{\mathbf{u}}_{v} \bar{\rho}_{v}\right)}{\partial t}+\nabla \cdot\left(\phi_{l} \overline{\mathbf{u}}_{l} \overline{\mathbf{u}}_{l} \bar{\rho}_{l}+\phi_{v} \overline{\mathbf{u}}_{v} \overline{\mathbf{u}}_{v} \bar{\rho}_{v}\right) \\
=-\nabla \cdot\left(\left\langle\overleftrightarrow{\boldsymbol{\sigma}}_{l}\right\rangle+\left\langle\overleftrightarrow{\boldsymbol{\sigma}}_{v}\right\rangle\right)-\nabla \cdot\left(\phi_{l} \stackrel{\leftrightarrow}{\boldsymbol{\tau}}_{\operatorname{Re}}^{(l)}+\phi_{v} \overleftrightarrow{\boldsymbol{\tau}}_{\operatorname{Re}}^{(v)}\right) \\
+\phi_{l} \overline{\mathbf{f}}_{l}+\phi_{v} \overline{\mathbf{f}}_{v}+\mathbf{f}_{l, i}+\mathbf{f}_{v, i}+\mathbf{f}_{l}^{\hat{\rho}}+\mathbf{f}_{v}^{\hat{\rho}} .
\end{gathered}
$$

For incompressible phases, the deviatoric stress tensor for phase $k$ is given by $\stackrel{\leftrightarrow}{\tau}_{k}=\mu_{k}\left(\nabla \mathbf{u}_{k}+\nabla \mathbf{u}_{k}^{T}\right)$, then the averaged stress tensor reads

$$
\left\langle\overleftrightarrow{\boldsymbol{\sigma}}_{k}\right\rangle=\phi_{k} \bar{P}_{k} \stackrel{\leftrightarrow}{\boldsymbol{I}}-\phi_{k} \stackrel{\leftrightarrow}{\boldsymbol{\tau}}_{k}-\stackrel{\leftrightarrow}{\boldsymbol{\tau}}_{k},
$$

where each tensor is given by

$$
\begin{gathered}
\stackrel{\leftrightarrow}{\boldsymbol{\tau}}_{k}=\bar{\mu}_{k}\left(\nabla \overline{\mathbf{u}}_{k}+\nabla \overline{\mathbf{u}}_{k}^{T}\right), \\
\stackrel{\leftrightarrow}{\boldsymbol{\tau}}_{k}=\frac{\bar{\mu}_{k}}{V} \int_{A_{k}}\left(\hat{\mathbf{u}}_{k, i} \mathbf{n}_{k}+\left(\hat{\mathbf{u}}_{k, i} \mathbf{n}_{k}\right)^{T}\right) d A_{k} \\
+\left(\left\langle\hat{\mu}_{k} \nabla \hat{\mathbf{u}}_{k}\right\rangle^{k}+\left\langle\hat{\mu}_{k} \nabla \hat{\mathbf{u}}_{k}^{T}\right\rangle^{k}\right) .
\end{gathered}
$$

Here, $\bar{P}_{k}$ is the average pressure in phase $k$ and $\hat{\mathbf{u}}_{k, i}$ represents a velocity fluctuation at the $k$ side of the interface and $\overleftrightarrow{\boldsymbol{I}}$ is the identity matrix. It is important to note that Eq. (29) is exact and no assumptions or special models have been used in its 
derivation. For constant bulk properties, the fluctuating force $\mathbf{f}_{k}^{\hat{\rho}}$ disappears. Furthermore, if the velocity fluctuations at the interface are random, the fluctuating tensor $\hat{\boldsymbol{\tau}}_{k}$ can be made vanished by taking an ensemble average of it. By defining mixture averaged quantities as $\bar{\Phi}=\phi_{l} \bar{\Phi}_{l}+\phi_{v} \bar{\Phi}_{v}$, Eq. (29) can be written in a simpler form as

$$
\begin{aligned}
& \frac{\partial\left(\phi_{l} \overline{\mathbf{u}}_{l} \bar{\rho}_{l}+\phi_{v} \overline{\mathbf{u}}_{v} \bar{\rho}_{v}\right)}{\partial t}+\nabla \cdot\left(\phi_{l} \overline{\mathbf{u}}_{l} \overline{\mathbf{u}}_{l} \bar{\rho}_{l}+\phi_{v} \overline{\mathbf{u}}_{v} \overline{\mathbf{u}}_{v} \bar{\rho}_{v}\right) \\
& =-\nabla \bar{P}+\nabla \cdot\left(\stackrel{\overline{\boldsymbol{\tau}}}{-\stackrel{\boldsymbol{\tau}}{\mathrm{Te}}_{\mathrm{Re}}}\right)+\overline{\mathbf{f}}+\mathbf{f}_{l v}^{\sigma},
\end{aligned}
$$

where $\mathbf{f}_{l v}^{\sigma}=\mathbf{f}_{l, i}+\mathbf{f}_{v, i}$ is the net interfacial force between the liquid and vapor. Although Eq. (33) is written in a more compact manner than Eq. (29), an important problem remains: Equation (33) still requires two velocity fields. As mentioned earlier, in the presence of phase change with unequal densities, the velocity of the two phases in contact cannot be equal. In the onedimensional case depicted in Fig. 1, the velocity of the vapor is zero and we were left with only one unknown velocity field. In a more general situation, both velocity fields are not null and, therefore, it is necessary to find a way of expressing Eq. (33) in terms of the mixture velocity $\overline{\mathbf{u}}$. This can be accomplished by combining Eq. (24) with the definition of the average velocity jump $\Delta \overline{\mathbf{u}}=\overline{\mathbf{u}}_{l}-\overline{\mathbf{u}}_{v}$, leading to

$$
\left(\begin{array}{c}
\overline{\mathbf{u}}_{l} \\
\overline{\mathbf{u}}_{v}
\end{array}\right)=\left(\begin{array}{c}
\overline{\mathbf{u}}+\phi_{v} \Delta \overline{\mathbf{u}} \\
\overline{\mathbf{u}}-\phi_{l} \Delta \overline{\mathbf{u}}
\end{array}\right) .
$$

With this, each term within the parentheses in the left-hand side of Eq. (33) can be expressed as

$$
\phi_{l} \overline{\mathbf{u}}_{l} \bar{\rho}_{l}+\phi_{v} \overline{\mathbf{u}}_{v} \bar{\rho}_{v}=\bar{\rho} \overline{\mathbf{u}}+\phi_{l} \phi_{v} \Delta \overline{\mathbf{u}}\left(\bar{\rho}_{l}-\bar{\rho}_{v}\right)
$$

and

$$
\begin{aligned}
\phi_{l} \overline{\mathbf{u}}_{l} \overline{\mathbf{u}}_{l} \bar{\rho}_{l}+\phi_{v} \overline{\mathbf{u}}_{v} \overline{\mathbf{u}}_{v} \bar{\rho}_{v} & \\
= & \bar{\rho} \overline{\mathbf{u}} \overline{\mathbf{u}}+\phi_{l} \phi_{v}\left(\overline{\mathbf{u}} \Delta \overline{\mathbf{u}}+(\overline{\mathbf{u}} \Delta \overline{\mathbf{u}})^{T}\right)\left(\bar{\rho}_{l}-\bar{\rho}_{v}\right) \\
& +\phi_{l} \phi_{v}\left(\phi_{v} \bar{\rho}_{l}+\phi_{l} \bar{\rho}_{v}\right) \Delta \overline{\mathbf{u}} \Delta \overline{\mathbf{u}} .
\end{aligned}
$$

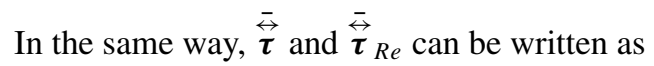

$$
\begin{aligned}
\stackrel{\leftrightarrow}{\boldsymbol{\tau}}= & \frac{1}{2} \bar{\mu}\left(\nabla \overline{\mathbf{u}}+\nabla \overline{\mathbf{u}}^{T}\right)+\frac{1}{2}\left[\phi_{l} \bar{\mu}_{l}\left(\nabla \overline{\mathbf{u}}_{l}+\nabla \overline{\mathbf{u}}_{l}^{T}\right)\right. \\
& \left.+\phi_{v} \bar{\mu}_{v}\left(\nabla \overline{\mathbf{u}}_{v}+\nabla \overline{\mathbf{u}}_{v}^{T}\right)-\bar{\mu}\left(\nabla \overline{\mathbf{u}}+\nabla \overline{\mathbf{u}}^{T}\right)\right]
\end{aligned}
$$

and

$$
\overline{\boldsymbol{\tau}}_{R e}=\bar{\rho}\langle\hat{\mathbf{u}} \hat{\mathbf{u}}\rangle+\left[\phi_{l} \bar{\rho}_{l}\left\langle\hat{\mathbf{u}}_{l} \hat{\mathbf{u}}_{l}\right\rangle^{l}+\phi_{v} \bar{\rho}_{v}\left\langle\hat{\mathbf{u}}_{v} \hat{\mathbf{u}}_{v}\right\rangle^{v}-\bar{\rho}\langle\hat{\mathbf{u}} \hat{\mathbf{u}}\rangle\right],
$$

where $\hat{\mathbf{u}}=\phi_{l} \hat{\mathbf{u}}_{l}+\phi_{v} \hat{\mathbf{u}}_{v}$. Since all the terms containing $\phi_{l} \phi_{v}$ in Eqs. (35) and (36) and the terms inside the square brackets in Eqs. (37) and (38) are zero outside the diffuse interface, they will not create a velocity jump or a net force between the two phases. In fact, these terms will only change the dynamic pressure distribution inside the diffuse interface, keeping intact the pressure jump due to recoil or surface tension effects. For laminar flow, the Reynolds-stress-like tensor could be nonzero only within the diffuse interface, due to fluctuations in the velocity field induced by the phase change (not by a turbulent flow). Based on these facts, we can express the mixture momentum equation in its final form as

$$
\begin{aligned}
\frac{\partial(\bar{\rho} \overline{\mathbf{u}})}{\partial t}+\nabla \cdot(\bar{\rho} \overline{\mathbf{u}} \overline{\mathbf{u}})= & -\nabla \bar{P}^{*}+\frac{1}{2} \nabla \cdot\left(\bar{\mu}\left(\nabla \overline{\mathbf{u}}+\nabla \overline{\mathbf{u}}^{T}\right)\right) \\
& +\overline{\mathbf{f}}+\mathbf{f}_{l v}^{\sigma}-\nabla \cdot(\bar{\rho}\langle\hat{\mathbf{u}} \hat{\mathbf{u}}\rangle),
\end{aligned}
$$

where the term $\mathbf{u} u$ has been kept only for completeness. This term acquires a physical relevance only for turbulent two-phase flows. The modified pressure $\bar{P}^{*}$ has been defined by

$$
\begin{aligned}
\nabla \bar{P}^{*}= & \nabla \bar{P}+\frac{\partial\left(\phi_{l} \phi_{v} \Delta \overline{\mathbf{u}}\left(\bar{\rho}_{l}-\bar{\rho}_{v}\right)\right)}{\partial t} \\
& +\nabla \cdot\left(\phi_{l} \phi_{v}\left(\overline{\mathbf{u}} \Delta \overline{\mathbf{u}}+(\overline{\mathbf{u}} \Delta \overline{\mathbf{u}})^{T}\right)\left(\bar{\rho}_{l}-\bar{\rho}_{v}\right)\right) \\
& +\nabla \cdot\left(\phi_{l} \phi_{v}\left(\phi_{v} \bar{\rho}_{l}+\phi_{l} \bar{\rho}_{v}\right) \Delta \overline{\mathbf{u}} \Delta \overline{\mathbf{u}}\right) \\
& -\frac{1}{2} \nabla \cdot\left(\phi_{l} \bar{\mu}_{l}\left(\nabla \overline{\mathbf{u}}_{l}+\nabla \overline{\mathbf{u}}_{l}^{T}\right)+\phi_{v} \bar{\mu}_{v}\left(\nabla \overline{\mathbf{u}}_{v}+\nabla \overline{\mathbf{u}}_{v}^{T}\right)\right. \\
& \left.-\bar{\mu}\left(\nabla \overline{\mathbf{u}}+\nabla \overline{\mathbf{u}}^{T}\right)\right)+\nabla \cdot\left(\bar{\leftrightarrow}_{R e}-\bar{\rho}\langle\hat{\mathbf{u}} \hat{\mathbf{u}}\rangle\right) .
\end{aligned}
$$

For quasi-incompressible formulations, the pressure in the momentum equations does not correspond to a thermodynamic variable and, therefore, it must be interpreted as a dynamic quantity used to enforce mass conservation. Furthermore, the fact that, outside the diffuse interface, $\bar{P}=\bar{P}^{*}$ guarantees that the pressure jump, arising from surface tension and recoil effects, stays unaltered. To recover the original mixture pressure distribution inside the diffuse interface, we must solve Eq. (40). Nonetheless, the solution of this equation is unjustified for two reasons: (1) the velocity profile across the diffuse interface depends exclusively on the mass conservation equation (not on $\bar{P}$ or $\bar{P}^{*}$ ) and (2) the modified pressure satisfies the pressure jump.

\section{Phase-field equation}

Although Eq. (22) is enough to describe the evolution of the phase field, this equation is not expressed in terms of a mixture velocity, which makes it useful only when one of the two velocity fields is known. A more general equation can be obtained by substracting Eq. (22) for the liquid and vapor and expressing the bulk velocities in terms of the mixture velocity and velocity jump,

$$
\frac{\partial \phi_{l}}{\partial t}+\nabla \cdot\left(\phi_{l} \overline{\mathbf{u}}+\phi_{l} \phi_{v} \Delta \overline{\mathbf{u}}\right)=-\frac{\ddot{\Gamma}_{l}}{\rho_{l}} .
$$

This equation differs from the one derived from a siglefluid formulation Eq. (10b), where the new term $\phi_{l} \phi_{v} \Delta \overline{\mathbf{u}}$ balances the nonlinear effects arising from $\phi_{l} \overline{\mathbf{u}}$. By assuming incompressible phases $\nabla \cdot \overline{\mathbf{u}}_{l}=\nabla \cdot \overline{\mathbf{u}}_{v}=0$, the divergence of the velocity can be expressed as

$$
\nabla \cdot \overline{\mathbf{u}}=\Delta \overline{\mathbf{u}} \cdot \nabla \phi_{l} .
$$

Applying volume conservation $\phi_{l}+\phi_{v}=1$ and replacing Eq. (42) into Eq. (41) yields

$$
\frac{\partial \phi_{l}}{\partial t}+\nabla \cdot\left(\phi_{l} \overline{\mathbf{u}}\right)=-\frac{\ddot{\Gamma}_{l}}{\rho_{l}}+\psi \nabla \cdot \overline{\mathbf{u}},
$$

where

$$
\psi=2 \phi_{l}-1
$$

is another order parameter that varies between -1 and 1 . Since the term $\psi \nabla \cdot \overline{\mathbf{u}}$ eliminates the spurious effects arising 
from nonlinear terms in Eq. (43), the only further requirement to propagate the phase field with an invariant profile is an even distribution of the mass evaporation rate across the diffuse interface. If the mass evaporation rate is not symmetric respect to $\phi_{l}=0.5$, then an additional conservative sharpening equation is required to enforce a desired profile.

\section{Averaged energy equation}

Neglecting viscous dissipation, the averaged energy equation reads

$$
\left\langle\frac{\partial\left(\rho_{k} h_{k}\right)}{\partial t}+\nabla \cdot\left(\mathbf{u}_{k} \rho_{k} h_{k}\right)\right\rangle=-\left\langle\nabla \cdot \mathbf{q}_{k}+S_{k}\right\rangle .
$$

Using the modified nomenclature for averaged variables, the energy equation for phase $k$ is given by

$$
\begin{aligned}
& \frac{\partial\left(\phi_{k} \bar{\rho}_{k} \bar{h}_{k}\right)}{\partial t}+\nabla \cdot\left(\phi_{k} \overline{\mathbf{u}}_{k} \bar{\rho}_{k} \bar{h}_{k}\right) \\
& =-\nabla \cdot\left(\phi_{k} \overline{\mathbf{q}}_{k}\right)+\phi_{k} \bar{S}_{k}-\frac{1}{V} \int_{A_{k}}\left(\mathbf{q}_{k}+\left(\mathbf{u}_{k}-\mathbf{u}_{i}\right) \rho_{k} h_{k}\right) \\
& \quad \cdot \mathbf{n}_{k} d A_{k}-\nabla \cdot\left(\phi_{k}\left\langle\hat{\mathbf{p}}_{k} \hat{\rho}_{k}\right\rangle\right)-\frac{\partial}{\partial t}\left(\phi_{k}\left\langle\hat{\rho}_{k} \hat{h}_{k}\right\rangle^{k}\right) \\
& \quad-\nabla \cdot\left(\phi_{k} \bar{h}_{k}\left\langle\hat{\mathbf{u}}_{k} \hat{\rho}_{k}\right\rangle^{k}\right) .
\end{aligned}
$$

Considering constant bulk densities and neglecting enthalpy fluctuations, the addition of the energy equation for the liquid and vapor yields

$$
\begin{aligned}
& \frac{\partial}{\partial t}\left(\phi_{l} \rho_{l} \bar{h}_{l}+\phi_{v} \rho_{v} \bar{h}_{v}\right)+\nabla \cdot\left(\phi_{l} \overline{\mathbf{u}}_{l} \rho_{l} \bar{h}_{l}+\phi_{v} \overline{\mathbf{u}}_{v} \rho_{v} \bar{h}_{v}\right) \\
& \quad=-\nabla \cdot\left(\phi_{l} \overline{\mathbf{q}}_{l}+\phi_{v} \overline{\mathbf{q}}_{v}\right)+\phi_{l} \bar{S}_{l}+\phi_{v} \bar{S}_{v}
\end{aligned}
$$

where the energy balance at the sharp interface $\mathbf{q}_{l}-\mathbf{q}_{v}+$ $\dot{\Gamma}_{l}\left(h_{l}-h_{v}\right) \mathbf{n}_{l}=0$ has been implicitly used. In line with the sigle-fluid derivation, we can define a mass-weighted average enthalpy as $\bar{h}=\left(\phi_{l} \bar{\rho}_{l} \bar{h}_{l}+\phi_{v} \bar{\rho}_{v} \bar{h}_{v}\right) / \bar{\rho}$. This allows us to express Eq. (47) in terms of the mixture velocity as

$$
\begin{aligned}
& \frac{\partial(\bar{\rho} \bar{h})}{\partial t}+\nabla \cdot(\bar{\rho} \overline{\mathbf{u}} \bar{h})+\nabla \cdot\left(\phi_{l} \phi_{v} \Delta \overline{\mathbf{u}}\left(\rho_{l} \bar{h}_{l}-\rho_{v} \bar{h}_{v}\right)\right) \\
& \quad=-\nabla \cdot\left(\phi_{l} \overline{\mathbf{q}}_{l}+\phi_{v} \overline{\mathbf{q}}_{v}\right)+\phi_{l} \bar{S}_{l}+\phi_{v} \bar{S}_{v} .
\end{aligned}
$$

By replacing Eq. (23) into Eq. (48) and defining a massweighted averaged heat capacity at constant pressure as $\bar{c}_{P}=$ $\left(\phi_{l} \bar{c}_{P l}+\phi_{v} \bar{c}_{P v}\right) / \bar{\rho}$, we arrive at

$$
\begin{aligned}
& \bar{\rho} \bar{c}_{P}\left(\frac{\partial T}{\partial t}+\overline{\mathbf{u}} \cdot \nabla T\right)+\left(\rho_{l} c_{P l}-\rho_{v} c_{P v}\right) \phi_{l} \phi_{v} \Delta \overline{\mathbf{u}} \cdot \nabla T \\
& \quad=-\nabla \cdot \overline{\mathbf{q}}-L \ddot{\Gamma}_{l}+\bar{S}
\end{aligned}
$$

where the mixture heat flux and source term are defined as $\overline{\mathbf{q}}=\phi_{l} \overline{\mathbf{q}}_{l}+\phi_{v} \overline{\mathbf{q}}_{v}=-\bar{k} \nabla T-\mathbf{j}_{n}$ and $\bar{S}=\phi_{l} \bar{S}_{l}+\phi_{v} \bar{S}_{v}$. The additional current $\mathbf{j}_{n}$ was determined from an asymptotic analysis of the phase-field model and is the thermal counterpart of the antitrapping current derived by Karma [13] for phase transitions driven by solutal diffusion (see asymptotic analysis). If the phase field is given by the hyperbolic tangent profile in Eq. (3b), then the gradient of the phase field satisfies $\left|\nabla \phi_{l}\right|=\sqrt{2} \phi_{l} \phi_{v} / W$. Replacing this expression into Eq. (42) yields $\phi_{l} \phi_{v} \Delta \overline{\mathbf{u}}=-W / \sqrt{2}(\nabla \cdot \overline{\mathbf{u}}) \mathbf{n}_{l}$. This result leads us to the final form of the energy equation

$$
\begin{aligned}
\bar{\rho} \bar{c}_{P}\left(\frac{\partial T}{\partial t}+\overline{\mathbf{u}} \cdot \nabla T\right)= & -\nabla \cdot \overline{\mathbf{q}}-L \ddot{\Gamma}_{l}+\bar{S}+\left(\rho_{l} c_{P l}-\rho_{v} c_{P v}\right) \\
& \times \frac{W}{\sqrt{2}}(\nabla \cdot \overline{\mathbf{u}}) \mathbf{n}_{l} \cdot \nabla T
\end{aligned}
$$

where the normal vector is defined as $\mathbf{n}_{l}=-\nabla \phi_{l} /\left|\nabla \phi_{l}\right|$. The last term in the right-hand side corrects the nonlinear effects in the energy equation arising from evaporation or condensation and vanishes outside the diffuse interface. For systems with high latent heat and density ratio (i.e., water at 1 bar), this term is fundamental to maintain the energy balance within the diffuse interface. Without it, no convergence to the sharp-interface limit can be achieved. A similar term was proposed by Hardt and Wondra [30] in their derivation of an evaporation model in the framework of volume-of-fluids modeling. Nonetheless, the evaporation rate presented by these authors is based on a microscopic description of the interface, where the rate of attachment and detachment of atoms (or molecules) depends on a single parameter, known as the evaporation coefficient, whose experimental values vary widely. In addition, the molecular theory used to derive the expression for the evaporation coefficient assumes ideal gas behavior in the vapor phase, which is far from the reality of a real gas close to the liquid vapor interface. For systems with a density ratio close to 1 , the divergence of the velocity field approaches to zero and this term becomes negligible compared to the release of absorption of latent heat. Keep in mind that Eq. (49) is a general expression for the energy equation, whereas Eq. (50) requires a phase field given by the hyperbolic tangent to be valid.

\section{SURFACE TENSION FORCE}

In the derivation of the averaged momentum equation, a net force between the liquid and vapor arises naturally from the averaging procedure. This force, which is given by

$$
\mathbf{f}_{l v}^{\sigma}=-\frac{1}{V} \int_{A_{l}}\left[\stackrel{\leftrightarrow}{\sigma_{l}}-\stackrel{\leftrightarrow}{\sigma_{v}}+\mathbf{p}_{l}\left(\mathbf{u}_{l}-\mathbf{u}_{i}\right)-\mathbf{p}_{v}\left(\mathbf{u}_{v}-\mathbf{u}_{i}\right)\right] \cdot \mathbf{n}_{l} d A_{l},
$$

represents an average value of the forces acting on the sharp interface within the diffuse interface. To give a physical interpretation to this force, we must analyze the behavior of matter down to the atomic scale. In the derivation of the averaged equations, it was assumed that the diffuse interface was composed by two bulk phases coexisting and separated by a membranelike sharp interface, with a morphology changing at the atomic scale. Hence, the first logical question is to know whether is physically possible to have bulk phases within the diffuse interface? To answer this question, we have to define, first, what we understand for a bulk phase. In fluids, a bulk phase is characterized by the collective behavior of a large number of atoms or molecules, where thermodynamic variables, such as density and pressure, are isotropic (invariant on rotation of the coordinate system). When isotropy is lost, the rotational symmetry of the system is broken and, consequently, we cannot speak about a bulk phase. From molecular theories [31], it is well known that the Cauchy stress tensor, obtained 
by averaging the interatomic interactions within the transition region, is not isotropic. Thus, this is the first indication that the state of matter, within a real interface, cannot be associated to any of the bulk phases in contact with the interface. Nowadays, almost everybody is aware of the four states of matter (solid, liquid, vapor, and plasma), but can we define a different state for atoms or molecules within an interface? At relatively low temperatures, the total volume occupied by an interface is very low, but at temperatures close to the critical point, interfaces get much thicker and, therefore, occupy a large volume. At the critical temperature, it is usually said that we do not have neither a liquid, nor a gas, but just a supercritical fluid. In essence, a supercritical fluid is another state of matter, just as is a superfluid or a superconductor material. Thus, a certain state of matter has a specific finger print, characterized by more than just the usual thermodynamic variables (temperature, density, and pressure). We would not notice the existence of a superconductor, if its electrical resistance was not measured or its remarkable interaction with magnets was not observed. In that regard, perhaps a fluid inside a diffuse interface is closer to a supercritical fluid than it is to the parent phases composing the interface. From all these arguments, I believe that is more evident now that the sharp interface at the atomic scale is just a fictitious entity used to derived the field equations, very much in the same way as is the control volume when deriving conservation laws.

To avoid entering the microscopic realm of interfacial fluids, it is easier to follow a more pragmatic path to determine the macroscopic surface tension force. The derivation provided in the following has been presented by several authors (an excellent derivation can be found in Ref. [32]), but I have decided to include it for the completeness of the article and to explain some details that are usually omitted in such derivations. From a mechanistic point of view, the surface tension is defined as the difference between the normal and tangential components of the stress tensor. In a bulk fluid, the stress tensor is spherically symmetric, but, as we move close to the interface, symmetry is broken and surface tension effects arise. From a thermodynamic view point, the surface tension is simply interpreted as the excess free energy associated to existence of the interface. The question now is in regard to how to reconcile these two interpretations into one formulation. It turns out that a thermodynamic derivation of the surface tension force includes both at the same time. To determine an expression for the surface tension, classic thermodynamics is not sufficient and a more general approach must be adopted. Following Cahn and Hilliard [33], we assume that the local value of the Helmholtz free energy can be decomposed into a homogeneous $f_{0}$ and inhomogeneous $f_{i h}$ parts. To obtain an expression for the inhomogeneous component, they considered that the free-energy density varies independently with a local order parameter and higher-order derivatives of it. For instance, when the system is described in terms of the density $\rho$, the free energy can be expanded in its Taylor's series around the homogeneous state as

$$
\begin{aligned}
f\left(\rho, \nabla \rho, \nabla^{2} \rho, \ldots\right)= & f_{0}(\rho)+\left(\frac{\partial f}{\partial \nabla \rho}\right)_{0} \nabla \rho+\left(\frac{\partial^{2} f}{\partial \nabla^{2} \rho}\right)_{0} \nabla^{2} \rho \\
& +\frac{1}{2}\left(\frac{\partial^{2} f}{\partial(\nabla \rho)^{2}}\right)_{0}|\nabla \rho|+\cdots
\end{aligned}
$$

Integrating this free energy over the entire volume and neglecting higher-order terms yields

$$
F=\int_{\Omega}\left\{f_{0}(\rho)+\frac{1}{2} \lambda(\rho, T, \ldots)|\nabla \rho|^{2}\right\} d \Omega,
$$

where the inhomogeneous coefficient $\lambda$ might depend on the density, temperature, concentration, and several other field variables. Although this coefficient could be derivable from rigorous molecular theories, the mathematical and theoretical difficulties posed by the many-body problem make it very difficult to reproduce accurately physical properties, such as surface tension. On the other hand, a phenomenological approach allows us to relate this coefficient directly to experimental values of the surface tension, with relatively small mathematical difficulties. To determine the inhomogeneous coefficient, we can make use of a phenomenological equation of state (EOS) that reproduces the phase diagram of a given system. Since we are interested in two-phase systems under quasiequilibrium conditions, the most important feature of the EOS is the correct representation of the stable phases in contact. This equation must relate the pressure with local values of density and temperature. From classic thermodynamics, the pressure can be related to the density, chemical potential $\mu$, and Helmholtz free energy through

$$
p_{0}=\mu \rho-f_{0}(\rho, T) .
$$

Figure 3 presents the variation of the Helmholtz free energy with the density and the meaning of the equilibrium pressure. The equilibrium densities are obtained from the common tangent line, whose slope is the chemical potential $\mu$. To have two phases under thermodynamic equilibrium, the pressure, chemical potential, and temperature must be equal for both phases. To find the density distribution that also satisfy the thermodynamic equilibrium, we must minimize the free-energy functional Eq. (53) with respect to the density, subjected to the mass conservation constraint. This requires the introduction of a Lagrange multiplier, which in this case is the chemical potential itself. Thus, the equilibrium density distribution must minimize the following functional:

$$
\Pi=\int_{\Omega}\left\{f_{0}(\rho)-\mu \rho+\frac{1}{2} \lambda|\nabla \rho|^{2}\right\} d \Omega .
$$

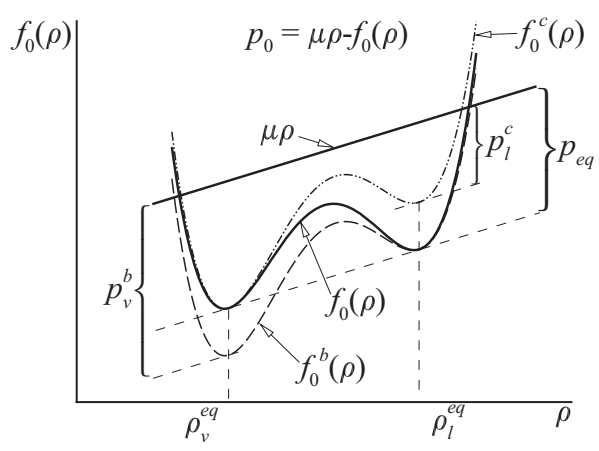

FIG. 3. Schematic representation of the thermodynamic pressure $p_{0}$, chemical potential $\mu$, and Helmholtz free-energy density $f_{0}$. The superindexes $b$ and $c$ represent the conditions for a curved interface during boiling and cavitation, respectively. 
Replacing the thermodynamic definition for the pressure Eq. (54), we have

$$
\Pi=\int_{\Omega}\left\{-p_{0}(\rho)+\frac{1}{2} \lambda|\nabla \rho|^{2}\right\} d \Omega .
$$

We now propose the following EOS:

$$
p_{0}(\rho, T)=p_{\text {eq }}(T)+A\left(\rho-\rho_{l}\right)^{2}\left(\rho-\rho_{v}\right)^{2},
$$

where $A$ is a proportionality constant whose magnitude is irrelevant for the current derivation. This EOS is not intended to accurately reproduce all the homogeneous equilibrium states of the bulk phases but only those close to the two-phase region. By using the definition for the mixture density, the pressure can be expressed as

$$
p_{0}\left(\phi_{l}, T\right)=p_{\text {eq }}(T)-A \Delta \rho^{4}(T) \phi_{l}^{2}\left(1-\phi_{l}\right)^{2} .
$$

For a flat interface, the density at each side of the interface correspond to the densities evaluated at the saturation temperature for a given equilibrium pressure; this is $\Delta \rho=\Delta \rho\left(T_{\text {sat }}\left(P_{\text {eq }}\right)\right)$. For a curved interface, the temperature of the system must increase (or decrease) to maintain an equilibrium condition and, therefore, the density of the bulk phases will change proportionally to the superheating (supercooling) required to compensate for the curvature effects. By linearizing the density difference, we have

$$
\Delta \rho=\Delta \rho_{\text {sat }}\left(1+\frac{1}{\Delta \rho_{\text {sat }}}\left(\frac{\partial \Delta \rho}{\partial T}\right)_{\text {sat }}\left(T-T_{\text {sat }}\right)\right)+O(\Delta T)^{2} .
$$

The second term in the parenthesis only becomes relevant for temperatures very close to the critical point. In fact, $\Delta \rho_{\text {sat }}^{-1}(\partial \Delta \rho / \partial T)_{\text {sat }} \approx-0.09 K^{-1}$ for water at $T=0.99 T_{c}$. Since the interface temperature is close to the saturation one, the second term in the parenthesis can be safely dropped, hence,

$$
p_{0}\left(\phi_{l}, T\right)=p_{\text {eq }}(T)-A \Delta \rho_{\text {sat }}^{4}\left(T_{\text {sat }}\right) \phi_{l}^{2}\left(1-\phi_{l}\right)^{2} .
$$

Substituting Eq. (60) into Eq. (56) and defining the inhomogeneous coefficient as $\lambda$ PF $=\lambda \Delta \rho_{\text {sat }}^{2}$, and the energy barrier as $\Delta f_{\max }=A \Delta \rho_{\text {sat }}^{4} / 16$, allows us to express Eq. (56) in terms of the phase field as

$$
\begin{aligned}
\Pi= & \int_{\Omega}\left\{-p_{\mathrm{eq}}(T)+16 \Delta f_{\max } \phi_{l}^{2}\left(1-\phi_{l}\right)^{2}\right. \\
& \left.+\frac{1}{2} \lambda_{\mathrm{PF}}\left|\nabla \phi_{l}\right|^{2}\right\} d \Omega .
\end{aligned}
$$

The equilibrium density distribution is achieved when the functional derivative of $\Pi$ respect to the phase field is zero. If the system is out of equilibrium, there will be a net driving force to evolve the phase field towards a distribution more energetically favorable. This driving force can be interpreted as a transformation potential whose form is

$$
\hat{\mu}=\frac{\delta \Pi}{\delta \phi_{l}}=-\frac{\partial p_{0}}{\partial \phi_{l}}-\lambda_{\mathrm{PF}} \nabla^{2} \phi_{l}-\frac{1}{2} \frac{\partial \lambda_{\mathrm{PF}}}{\partial \phi_{l}}\left|\nabla \phi_{l}\right|^{2} .
$$

From a rigorous thermodynamic point of view, this potential does not represent the real chemical potential but a departure from it. Therefore, if the transformation potential is zero, the system is under equilibrium and the chemical potential is the same everywhere. For an inhomogeneous coefficient independent of the phase field, the transformation potential reads

$$
-\hat{\mu}=\lambda_{\mathrm{PF}} \nabla^{2} \phi_{l}+16 \Delta f_{\max } 2 \phi_{l}\left(1-\phi_{l}\right)\left(2 \phi_{l}-1\right) .
$$

For a planar interface under equilibrium, the transformation potential is zero $(\hat{\mu}=0)$ and, therefore, the phase field that minimizes the functional Eq. (61) is the hyperbolic tangent profile given in Eq. (3b), where $W$ is related to the inhomogeneous coefficient and energy barrier by

$$
W=\sqrt{\lambda_{\mathrm{PF}} / 16 \Delta f_{\max }} .
$$

To relate the phase-field coefficients $\left(\lambda_{\mathrm{PF}}, \Delta f_{\max }\right)$ to the experimental value of the surface tension, we have to determine, first, the stress tensor under equilibrium conditions. For an inhomogeneous coefficient $\lambda_{\mathrm{PF}}$ that depends only on the phase field, we can multiply Eq. (62) by $\nabla \phi_{l}$ to arrive at

$$
\nabla \cdot\left(p_{0} \stackrel{\leftrightarrow}{\mathbf{I}}+\lambda_{\mathrm{PF}} \nabla \phi_{l} \nabla \phi_{l}-\frac{1}{2} \lambda_{\mathrm{PF}}\left|\nabla \phi_{l}\right|^{2} \stackrel{\leftrightarrow}{\mathbf{I}}\right)=-\hat{\mu} \nabla \phi_{l}
$$

From this, we define the equilibrium stress tensor (or capillary stress tensor) as

$$
\stackrel{\leftrightarrow}{\sigma}_{c}=\left(p_{0}+\frac{1}{2} \lambda_{\mathrm{PF}}\left|\nabla \phi_{l}\right|^{2}\right) \stackrel{\leftrightarrow}{\mathbf{I}}+\lambda_{\mathrm{PF}}\left|\nabla \phi_{l}\right|^{2}\left(\mathbf{n}_{v} \mathbf{n}_{v}-\stackrel{\leftrightarrow}{\mathbf{I}}\right),
$$

where the normal vector is defined as $\mathbf{n}_{v}=-\mathbf{n}_{l}=\nabla \phi_{l} /\left|\nabla \phi_{l}\right|$. To further clarify the meaning of this tensor, it is convenient to use a matrix representation,

$$
\overleftrightarrow{\boldsymbol{\sigma}}_{c}=\left(\begin{array}{ccc}
\sigma_{n n} & 0 & 0 \\
0 & \sigma_{t t} & 0 \\
0 & 0 & \sigma_{s s}
\end{array}\right) .
$$

In a curvilinear orthogonal coordinate system, the subindexes $n, t$, and $s$ correspond to the normal and tangential directions, respectively. The components of the capillary stress tensor are, thus, given by

$$
\begin{gathered}
\sigma_{n n}=p_{0}+\frac{1}{2} \lambda_{\mathrm{PF}}\left|\nabla \phi_{l}\right|^{2}, \\
\sigma_{t t}=p_{0}-\frac{1}{2} \lambda_{\mathrm{PF}}\left|\nabla \phi_{l}\right|^{2}, \\
\sigma_{s s}=p_{0}-\frac{1}{2} \lambda_{\mathrm{PF}}\left|\nabla \phi_{l}\right|^{2} .
\end{gathered}
$$

Using the mechanical definition of the surface tension (difference between the normal and tangential components of the stress tensor), the surface tension is given by

$$
\hat{\sigma}(r)=\lambda_{\mathrm{PF}}\left|\nabla \phi_{l}\right|^{2} \text {. }
$$

This is a distributed quantity, whose integral along the normal direction $r$ correspond to the macroscopic value of the surface tension, hence,

$$
\sigma=\int_{v}^{l} \hat{\sigma}(r) d r
$$

For a phase field following the hyperbolic tangent and a inhomogeneous coefficient independent of the order parameter $\phi_{l}$, the macroscopic surface tension is related to $\lambda_{\mathrm{PF}}$ through

$$
\sigma=\lambda_{\mathrm{PF}} \int_{v}^{l}\left(\frac{\partial \phi_{l}}{\partial r}\right)^{2} d r=\frac{\lambda_{\mathrm{PF}}}{4} \frac{2 \sqrt{2}}{3 W} .
$$


In the absence of curvature, it is possible to prove that the term $p_{0}+\lambda_{\mathrm{PF}}\left|\nabla \phi_{l}\right|^{2} / 2$ is constant across the diffuse interface (see Ref. [32]). Defining a modified pressure as $\tilde{p}=p_{0}+$ $\lambda_{\mathrm{PF}}\left|\nabla \phi_{l}\right|^{2} / 2$ and replacing the expression for the distributed surface tension Eq. (69) into Eq. (66), the capillary stress tensor reads

$$
\stackrel{\leftrightarrow}{\boldsymbol{\sigma}}_{c}=\tilde{p} \stackrel{\leftrightarrow}{\mathbf{I}}+\hat{\sigma}(r)\left(\mathbf{n}_{v} \mathbf{n}_{v}-\stackrel{\leftrightarrow}{\mathbf{I}}\right)
$$

Taking the divergence of this tensor leads to

$$
\nabla \cdot \overleftrightarrow{\sigma}_{c}=\nabla \tilde{p}+\kappa \hat{\sigma}(r) \mathbf{n}_{v}+\nabla \hat{\sigma}(r) \cdot\left(\mathbf{n}_{v} \mathbf{n}_{v}-\stackrel{\leftrightarrow}{\mathbf{I}}\right)
$$

If the surface tension depends on temperature, concentration, or any other field variable (other than the phase field), the third term in the right-hand side will produce a net force in the tangential direction. This force will induce a tangential flow, known as Marangoni convection. The second term in the right-hand side is the surface tension force, which is commonly modeled as $\kappa \sigma \nabla \phi_{l}$, where $\kappa$ is the local curvature and $\sigma$ the macroscopic surface tension. Equation (65) provides us with an expression for the divergence of the capillary stress tensor for an inhomogeneous coefficient dependent only on the phase field, but in a more general situation, this coefficient will also depend on the interface temperature, concentration, magnetic field, and so on. In general, the divergence of the stress tensor (net interfacial force) can be expressed as

$$
\mathbf{f}_{l v}^{\sigma}=-\nabla \cdot \stackrel{\leftrightarrow}{\sigma}_{c}=\hat{\mu} \nabla \phi_{l}-\nabla \hat{\sigma}(r) \cdot\left(\mathbf{n}_{v} \mathbf{n}_{v}-\stackrel{\leftrightarrow}{\mathbf{I}}\right)
$$

Combining the definition for $W$ Eq. (64) and Eq. (71), the phase-field parameters $\left(\lambda_{\mathrm{PF}}, \Delta f_{\max }\right)$ are related to the surface tension and $W$ through

$$
\left(\begin{array}{c}
\lambda_{\mathrm{PF}} \\
\Delta f_{\max }
\end{array}\right)=\left(\begin{array}{c}
4 \sigma W / I_{0} \\
\sigma / 4 W I_{0}
\end{array}\right)
$$

where

$$
I_{0}=\int_{-\infty}^{\infty}\left(\frac{\partial}{\partial \eta} \tanh \left(\frac{\eta}{\sqrt{2}}\right)\right)^{2} d \eta=\frac{2 \sqrt{2}}{3} .
$$

Replacing Eq. (75) into Eq. (63) yields the final expression for the potential entering the surface tension force

$$
\hat{\mu}=-\frac{4 \sigma}{W I_{0}}\left(W^{2} \nabla^{2} \phi_{l}+2 \phi_{l}\left(1-\phi_{l}\right)\left(2 \phi_{l}-1\right)\right) .
$$

\section{MASS EVAPORATION RATE}

For a nonconserved order parameter $\phi_{l}$, its temporal evolution due to phase change can be described the Allen-Cahn equation as

$$
\frac{\partial \phi_{l}}{\partial t}=-M \hat{\mu}
$$

where $M$ is a positive constant known as mobility coefficient. This equation represents a linear relaxation process of the phase field towards a more energetic favorable state. Expressing the Laplacian in curvilinear orthogonal coordinates, the transformation potential reads

$$
\begin{aligned}
\hat{\mu}= & -\frac{4 \sigma}{W I_{0}}\left(W^{2} \frac{\partial^{2} \phi_{l}}{\partial r^{2}}+2 \phi_{l}\left(1-\phi_{l}\right)\left(2 \phi_{l}-1\right)\right) \\
& -\frac{4 \sigma W}{I_{0}} \kappa\left|\nabla \phi_{l}\right| .
\end{aligned}
$$

Once again, $r$ is the normal direction pointing towards the liquid phase. If $\phi_{l}$ is given by the hyperbolic tangent Eq. (3b), then the big parenthesis in the right-hand side vanishes and the transformation potential simply reads

$$
\hat{\mu}=-\frac{4 \sigma W^{2}}{W I_{0}} \kappa\left|\nabla \phi_{l}\right| .
$$

Replacing Eq. (80) into Eq. (78) leads to

$$
\frac{\partial \phi_{l}}{\partial t}=\frac{W^{2}}{\tau} \kappa\left|\nabla \phi_{l}\right|,
$$

where the mobility coefficient $M$ is related to the relaxation time $\tau$ through $M=W I_{0} / 4 \sigma \tau$. This result shows that Eq. (78) cannot describe a curved interface under thermodynamic equilibrium. In the presence of curvature, $\partial \phi_{l} / \partial t \neq 0$ and, thus, no equilibrium can be attained. This phenomenon is known as curvature-driven motion and must be balanced by an additional function to describe properly a curved interface under equilibrium. This is done by introducing a new function into the free-energy functional

$$
\tilde{\Pi}=\int_{\Omega}\left\{-p_{0}\left(\phi_{l}, T\right)+\frac{1}{2} \lambda_{\mathrm{PF}}\left|\nabla \phi_{l}\right|^{2}+\gamma g\left(\phi_{l}\right) \Delta T\right\} d \Omega .
$$

The term $\gamma g\left(\phi_{l}\right) \Delta T$ accounts for the required superheating to balance the curvature effects. Hence, to equilibrate a curved interface, we must increase the temperature of the system above the saturation value corresponding to a planar interface, i.e., $T_{\text {sys }}^{\text {curved }}=T_{\text {sat }}^{\text {planar }}+\Delta T$. In addition, the function $g\left(\phi_{l}\right)$ must not alter the equilibrium values of the phase field, which can be achieved if $\partial g / \partial \phi_{l}=0$ for $\phi_{l}=\{0,1\}$. One function that is frequently used in phase-field models is

$$
g\left(\phi_{l}\right)=\left(2 \phi_{l}-1\right)-\frac{\left(2 \phi_{l}-1\right)^{3}}{3},
$$

thus, a new transformation potential is defined as

$$
\begin{aligned}
\tilde{\mu}= & -\frac{4 \sigma}{W I_{0}}\left(W^{2} \nabla^{2} \phi_{l}+2 \phi_{l}\left(1-\phi_{l}\right)\left(2 \phi_{l}-1\right)\right. \\
& \left.-\frac{\gamma W I_{0}}{\sigma} 2 \phi_{l}\left(1-\phi_{l}\right) \Delta T\right) .
\end{aligned}
$$

By using the identity $\left|\nabla \phi_{l}\right|=\sqrt{2} \phi_{l}\left(1-\phi_{l}\right) / W$ and expressing the Laplacian in orthogonal coordinates, we arrive at

$$
\tilde{\mu}=-\frac{4 \sigma}{I_{0}} \phi_{l}\left(1-\phi_{l}\right)\left(\kappa \sqrt{2}-\frac{2 \gamma I_{0}}{\sigma} \Delta T\right) .
$$

For a system under equilibrium, the transformation potential $\tilde{\mu}$ must be equal to 0 , therefore, in the presence of a curved interface, the system must be superheated by the amount

$$
\Delta T=\sigma \kappa / \gamma I_{0} \sqrt{2}
$$


By comparing this expression to a linearized version of the Clausius-Clapeyron equation

$$
\Delta T \approx \sigma \kappa T_{\mathrm{sat}}\left(\rho_{v}^{-1}-\rho_{l}^{-1}\right) / L_{\mathrm{sat}},
$$

we can relate $\gamma$ to physical quantities through

$$
\gamma=\frac{L_{\mathrm{sat}}}{\sqrt{2} I_{0} T_{\mathrm{sat}}\left(\rho_{v}^{-1}-\rho_{l}^{-1}\right)} .
$$

Since thermodynamic equilibrium requires a constant temperature (or superheating) throughout the system, Eq. (85) tells us that if the curvature changes along the interface, equilibrium cannot be achieved. Even in the case of a perfect spherical vapor bubble, the smallest variation on the bubble size or system temperature will initiate the uncontrollable growth or collapse of our hypothetical bubble due to evaporation or condensation respectively. To close the system of equations, the following evaporation (or condensation) rate per unit volume is proposed:

$$
\ddot{\Gamma}_{l}=\rho_{v} M \tilde{\mu} .
$$

This choice for the evaporation rate is based on the onedimensional evaporating system presented schematically in Figure 1, where the evaporation rate is proportional to the interface velocity and the density of the vapor. Although this expression might appear as arbitrary at first glance, its general validity was demonstrated with the solution of several problems in Sec. VIII. The asymptotic analysis of the phase-field equations (see Sec. V) revealed that the thermal capillary length associated to this model is given by

$$
d_{0}=\frac{\sigma T_{\mathrm{sat}} \rho_{v} c_{P v}^{\mathrm{sat}}\left(1-\rho_{v} / \rho_{l}\right)}{\left(\rho_{v} L_{\mathrm{sat}}\right)^{2}} .
$$

It is important not to confuse $d_{0}$ with the conventional capillary length associated with the formation of menisci during wetting phenomena $d_{c}=\sqrt{\sigma / g \Delta \rho}$, where $g$ is the Earth's gravitational acceleration and $\Delta \rho$ is the density difference between the liquid and gaseous phase. The thermal capillary length $d_{0}$ is a length scale associated to the curvature effects on the interface temperature. Phase-field simulations of the Mullins-Sekerka instability [34] have shown that accurate predictions of the interface dynamics can be achieved only for relatively low ratios between interface thickness and the solutal capillary length (analogous to the thermal capillary length when the phase change is controlled by a concentration field instead of the temperature). This sets a tight constraint on the system size that can be simulated with the current model. Figure 4 shows the variation of the thermal capillary length with temperature for saturated water, which lies on the nanometer scale. Hence, simulations of system sizes in the range of millimeters are completely ruled out. For nonisothermal systems, the superheating must be interpreted as a local quantity within the diffuse interface, which fulfills two functions: (1) balance of curvature effects and (2) generation of an imbalance in the transformation potential to produce a net evaporation (or condensation) rate. This means that the superheating can be split into two parts, one related to curvature effects $\Delta T_{\kappa}$ and another one related to the phase change $\Delta T_{t}$, thus, the total superheating is given by $\Delta T=\Delta T_{\kappa}+\Delta T_{t}$. This fact opens up a window for a solution to the small

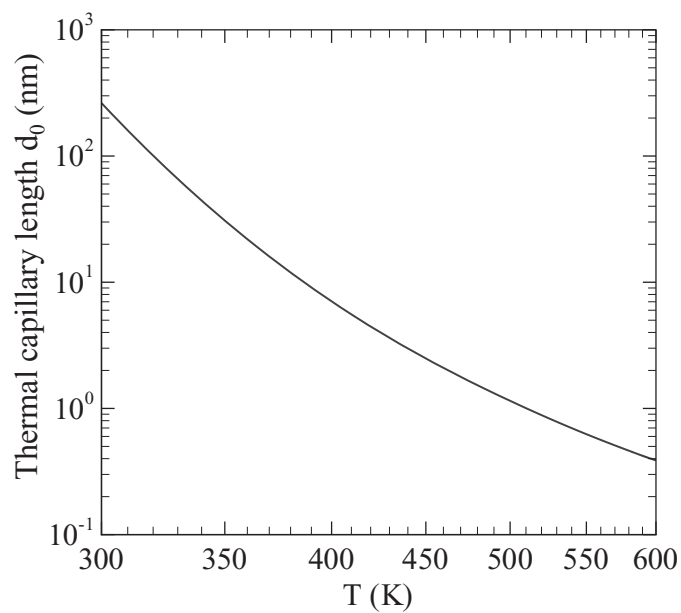

FIG. 4. Dependence of the thermal capillary length of water with the saturation temperature. Physical properties were obtained from the ASME steam table.

magnitude of the thermal capillary length: the elimination of the curvature from the transformation potential. By eliminating the curvature from the Laplacian of the phase field, we can also eliminate the superheating associated to it. See Sec. VI for a detailed discussion on how to eliminate the curvature from the phase-field equation.

\section{ASYMPTOTIC ANALYSIS}

To start the asymptotic analysis, it is convenient to write a summary with all the equations composing the model

Mass

$$
\boldsymbol{\nabla} \cdot \overline{\mathbf{u}}=-\left(\frac{1}{\rho_{l}}-\frac{1}{\rho_{v}}\right) \ddot{\Gamma}_{l},
$$

\section{Linear momentum}

$$
\begin{aligned}
& \frac{\partial(\bar{\rho} \overline{\mathbf{u}})}{\partial t}+\nabla \cdot(\bar{\rho} \overline{\mathbf{u}} \overline{)}) \\
& \quad=-\nabla \bar{P}^{*}+\frac{1}{2} \nabla \cdot\left(\bar{\mu}\left(\nabla \overline{\mathbf{u}}+\nabla \overline{\mathbf{u}}^{T}\right)\right)+\overline{\mathbf{f}}+\mathbf{f}_{l v}^{\sigma},
\end{aligned}
$$

Energy

$$
\begin{aligned}
& \bar{\rho} \bar{c}_{P}\left(\frac{\partial T}{\partial t}+\overline{\mathbf{u}} \cdot \nabla T\right) \\
& \quad=-\nabla \cdot \overline{\mathbf{q}}-L \ddot{\Gamma}_{l}+\bar{S}+\left(\rho_{l} c_{P l}-\rho_{v} c_{P v}\right) \frac{W}{\sqrt{2}}(\nabla \cdot \overline{\mathbf{u}}) \mathbf{n}_{l} \cdot \nabla T,
\end{aligned}
$$

Phase field

$$
\frac{\partial \phi_{l}}{\partial t}+\nabla \cdot\left(\phi_{l} \overline{\mathbf{u}}\right)=-\frac{\ddot{\Gamma}_{l}}{\rho_{l}}+\psi \nabla \cdot \overline{\mathbf{u}},
$$

Evaporation rate

$$
\begin{aligned}
\ddot{\Gamma}_{l}= & -\frac{\rho_{v}}{\tau}\left(W^{2} \nabla^{2} \phi_{l}+2 \phi_{l}\left(1-\phi_{l}\right)\left(2 \phi_{l}-1\right)\right. \\
& \left.-\frac{\gamma W I_{0}}{\sigma} 2 \phi_{l}\left(1-\phi_{l}\right) \Delta T\right) .
\end{aligned}
$$


The asymptotic analysis of these equations is quite complex, but it can be simplified by using an equivalent set of equations. Without loss of generality, this can be done by considering the one-dimensional situation depicted in Fig. 1. In that case, the velocity of the vapor phase is zero and the velocity of the liquid phase can be obtained by direct integration of the mass conservation equation. This allows us to eliminate the momentum equation from the analysis. By setting the vapor velocity to zero, the phase-field model can be written as

Mass

$$
\nabla \cdot\left(\overline{\mathbf{u}}_{l} \phi_{l}\right)=-\left(\frac{1}{\rho_{l}}-\frac{1}{\rho_{v}}\right) \ddot{\Gamma}_{l},
$$

Energy

$$
\bar{\rho} \bar{c}_{P} \frac{\partial T}{\partial t}+\rho_{l} c_{P l} \overline{\mathbf{u}}_{l} \phi_{l} \cdot \nabla T=-\nabla \cdot \overline{\mathbf{q}}-L \ddot{\Gamma}_{l}+\bar{S},
$$

Phase field

$$
\frac{\partial \phi_{l}}{\partial t}=-\frac{\ddot{\Gamma}_{l}}{\rho_{v}},
$$

where the evaporation rate is still given by Eq. (95). When considering an interface under local thermodynamic equilibrium, it is possible to express the variation of the temperature within the diffuse interface in terms of the average interface velocity,

$$
\frac{\partial T}{\partial t}=-\overline{\mathbf{u}}_{i} \cdot \nabla T .
$$

This allows us to express the energy equation in the following form:

$$
\begin{aligned}
& \rho_{v} \tilde{c_{P}} \frac{\partial T}{\partial t}+c_{P l} \phi_{l}\left[\rho_{l}\left(\overline{\mathbf{u}}_{l}-\overline{\mathbf{u}}_{i}\right)+\rho_{v} \overline{\mathbf{u}}_{i}\right] \cdot \nabla T \\
& \quad=-\nabla \cdot \overline{\mathbf{q}}-L \ddot{\Gamma}_{l}+\bar{S},
\end{aligned}
$$

where the volume-averaged heat capacity is defined as $\tilde{c}_{P}=$ $c_{P l} \phi_{l}+c_{P v} \phi_{v}$. Mass continuity requires $\rho_{l}\left(\overline{\mathbf{u}}_{l}-\overline{\mathbf{u}}_{i}\right)+\rho_{v} \overline{\mathbf{u}}_{i}=$ 0 for $\overline{\mathbf{u}}_{v}=0$ and, thus, the energy equation reads

$$
\rho_{v} \tilde{c_{P}} \frac{\partial T}{\partial t}=-\nabla \cdot \overline{\mathbf{q}}-L \ddot{\Gamma}_{l}+\bar{S}
$$

With the elimination of the velocity field from the energy equation, the phase-field model reduces to only two equations, facilitating its asymptotic analysis. By replacing the definition of the mixture heat flux $\overline{\mathbf{q}}=-\bar{k} \nabla T-\mathbf{j}_{n}$, the reduced model is given by

Phase field

$$
\begin{aligned}
\frac{\partial \phi_{l}}{\partial t}= & \frac{1}{\tau}\left(W^{2} \nabla^{2} \phi_{l}+2 \phi_{l}\left(1-\phi_{l}\right)\left(2 \phi_{l}-1\right)\right. \\
& \left.-\frac{\gamma W I_{0}}{\sigma} 2 \phi_{l}\left(1-\phi_{l}\right) \Delta T\right)
\end{aligned}
$$

Energy

$$
\rho_{v} \tilde{c_{P}} \frac{\partial T}{\partial t}=\nabla \cdot\left(\bar{k} \nabla T+\mathbf{j}_{n}\right)+\rho_{v} L \frac{\partial \phi_{l}}{\partial t}+\bar{S},
$$

where the phenomenological current $\mathbf{j}_{n}$ is defined by

$$
\mathbf{j}_{n}=a(\psi) W L \ddot{\Gamma}_{l} \mathbf{n}_{l} .
$$

This current is analogous to that proposed originally by Karma [13] when studying dendritic growth. This current is primarily needed to cancel out spurious effects, arising from the steep variation of the thermal conductivity, across the diffuse interface. To facilitate the asymptotic analysis, it is convenient to use a linearized version of the enthalpy around the saturation temperature,

$$
h_{j}=h_{j}^{\mathrm{sat}}+\left(\frac{\partial h_{j}}{\partial T}\right)_{\mathrm{sat}}\left(T-T_{\mathrm{sat}}\right) .
$$

With this definition, the volume-weighted averaged heat capacity and latent heat read

$$
\begin{gathered}
\tilde{c_{P}}=\frac{\partial h_{l}}{\partial T} \phi_{l}+\frac{\partial h_{v}}{\partial T} \phi_{v}=c_{l}^{\mathrm{sat}} \phi_{l}+c_{v}^{\mathrm{sat}} \phi_{v}, \\
L=L_{\mathrm{sat}}-\Delta c_{P}^{\mathrm{sat}} \Delta T,
\end{gathered}
$$

where the heat capacity difference $\Delta c_{P}^{\mathrm{sat}}=c_{P l}^{\mathrm{sat}}-c_{P v}^{\mathrm{sat}}$ and latent heat $L_{\mathrm{sat}}$ are evaluated at the saturation temperature. The local superheating is represented by $\Delta T=T-T_{\text {sat }}$. By using a linearized enthalpy and measuring time in units of $l_{c}^{2} / D_{v}$ and length in units of $l_{c}$, we can express the energy and phase-field equations in dimensionless form as

Energy

$$
\begin{aligned}
& \partial_{\breve{t}}\left(\left[1+(\varpi-1) \phi_{l}\right] \theta-\phi_{l}\right) \\
& \quad=\breve{\nabla} \cdot\left(q \breve{\nabla} \theta+a\left(\phi_{l}\right) \epsilon(1-(\varpi-1) \theta) \partial_{\breve{t}} \phi_{l} \mathbf{n}_{v}\right),
\end{aligned}
$$

\section{Phase field}

$$
\alpha \epsilon^{2} \partial_{\breve{t}} \psi=\epsilon^{2} \breve{\nabla}^{2} \psi+\left(1-\psi^{2}\right)(\psi-\epsilon \breve{\gamma} \theta),
$$

where the following dimensionless quantities have been defined:

$$
\begin{aligned}
& \theta=\frac{c_{P v}^{\mathrm{sat}} \Delta T}{L_{\mathrm{sat}}}, \quad \epsilon=\frac{W}{l_{c}}, \quad \alpha=\frac{\tau D_{v}}{W^{2}}, \quad \varpi=\frac{c_{P l}^{\mathrm{sat}}}{c_{P v}^{\mathrm{sat}}} \\
& q=\frac{k(\phi)}{k_{v}}, \quad D_{v}=\frac{k_{v}}{\rho_{v} c_{P v}^{\mathrm{sat}}}, \quad \breve{\gamma}=\frac{\gamma I_{0} L_{\mathrm{sat}} l_{c}}{\sigma c_{P v}^{\mathrm{sat}}} .
\end{aligned}
$$

For a diffuse interface with constant thickness, the temporal derivative can be expressed in terms of the interface velocity as $\partial_{\breve{t}}=-\breve{u}_{i} \partial_{\breve{r}}$, where $\breve{r}$ is the dimensionless normal direction. Following the standard asymptotic analysis $[13,16]$, we divide the system into two regions: (1) the inner region which considers the inside of the diffuse interface and (2) the outer region representing the bulk phases. By making use of a three-dimensional curvilinear orthogonal system with a stretched radial coordinate $\eta=r / W=\breve{r} / \epsilon$ (see Appendix B), we can rewrite Eqs. (108) and (109) as

\section{Energy}

$$
\begin{aligned}
& \partial_{\eta}\left(q \partial_{\eta} \theta\right)+\epsilon\left\{\breve{\kappa} q \partial_{\eta} \theta-\partial_{\eta}\left[a\left(\phi_{l}\right)(1-(\varpi-1)) \breve{u}_{i} \partial_{\eta} \phi_{l}\right]\right. \\
& \left.\quad+\breve{u}_{i}\left[\left(1+(\varpi-1) \phi_{l}\right) \theta-\phi_{l}\right]\right\} \\
& \quad+\epsilon^{2}\left\{\partial_{s}\left(q \partial_{s} \theta\right)-\frac{1}{2} \breve{\kappa}^{2} \eta q \partial_{\eta} \theta-a(\psi) \breve{\kappa} \breve{u}_{i} \partial_{\eta} \phi_{l}\right\} \\
& \quad+O\left(\epsilon^{3}\right)=0
\end{aligned}
$$


Phase field

$$
\begin{gathered}
\partial_{\eta \eta} \psi+\left(1-\psi^{2}\right) \psi+\epsilon\left(\breve{\kappa}+\alpha \breve{u}_{i}-\sqrt{2} \breve{\gamma} \theta\right) \partial_{\eta} \psi \\
-\frac{1}{2} \epsilon^{2} \breve{\kappa}^{2} \eta \partial_{\eta} \psi+O\left(\epsilon^{3}\right)=0 .
\end{gathered}
$$

For the outer region, a stretched coordinate is not required and, thus, the energy equation reads

$$
\begin{aligned}
& q^{ \pm} \partial_{\breve{r} \breve{r}} \hat{\theta}+q^{ \pm} \partial_{\breve{r}} \hat{\theta} \breve{\kappa}\left(1-\frac{\breve{\kappa}}{2} \breve{r}\right)+q^{ \pm} \partial_{s s} \hat{\theta}\left(1-2 \breve{r} \breve{\kappa}_{s}\right) \\
& +\breve{u}_{i}\left[1+(\varpi-1) \phi^{ \pm}\right] \partial_{\breve{r}} \hat{\theta}=0,
\end{aligned}
$$

where $\breve{\kappa}_{s}$ is the curvature along the $s$ direction and the superindex \pm represents the bulk liquid $(+)$ and bulk vapor $(-)$.

To analyze the convergence of this model towards the sharpinterface limit, we expand both fields in the inner region in powers of $\epsilon$ as

$$
\begin{aligned}
\phi_{l} & =(1+\psi) / 2 \\
\psi & =\psi_{0}+\epsilon \psi_{1}+\epsilon^{2} \psi_{2}+\cdots \\
\theta & =\theta_{0}+\epsilon \theta_{1}+\epsilon^{2} \theta_{2}+\cdots .
\end{aligned}
$$

A similar expansion is performed for the outer temperature field $\hat{\theta}=\hat{\theta}_{0}+\epsilon \hat{\theta}_{1}+\epsilon^{2} \hat{\theta}_{2}+\cdots$. Since the phase field outside the diffuse interface is constant, we simply have $\psi^{ \pm}= \pm 1$. Any function depending on the phase field is expanded in the inner region as

$$
\begin{aligned}
f(\psi)= & f^{(0)}+f_{\psi}^{(0)}\left(\epsilon \psi_{1}+\epsilon^{2} \psi_{2}+\cdots\right) \\
& +\frac{1}{2} f_{\psi \psi}^{(0)}\left(\epsilon \psi_{1}+\epsilon^{2} \psi_{2}+\cdots\right)^{2}+\cdots,
\end{aligned}
$$

where $f^{(0)} \equiv f\left(\psi_{0}\right)$ and $f_{\psi}^{(0)} \equiv(\partial f / \partial \psi)_{\psi=\psi_{0}}$. The matching conditions can be readily obtained by equating order by order the inner and outer fields (and their derivatives) at the matching points (see Fig. 5), hence,

$$
\begin{gathered}
\lim _{\eta \rightarrow \pm \infty} \psi_{0}=\left.\hat{\psi}_{0}\right|_{ \pm}= \pm 1 \\
\lim _{\eta \rightarrow \pm \infty} \partial_{\eta} \psi_{j}=0 .
\end{gathered}
$$

To find the matching conditions for the temperature, we assume that the outer temperature field extends up to the interface $(\eta=0)$ and then expand it in a Taylor series centered at the

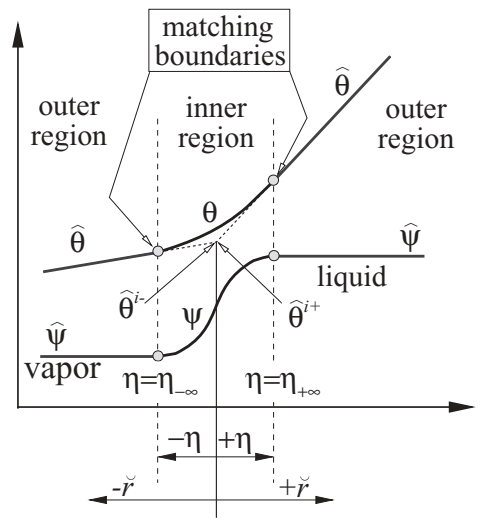

FIG. 5. Asymptotic schematic. interface, thus, the outer temperature reads

$$
\begin{aligned}
\hat{\theta} & =\hat{\theta}^{i \pm}+\left.\partial_{\breve{r}} \hat{\theta}\right|_{i \pm} \breve{r}+\left.\frac{1}{2} \partial_{\breve{r} \breve{r}} \hat{\theta}\right|_{i \pm} \breve{r}^{2}+\cdots \\
& =\hat{\theta}^{i \pm}+\left.\epsilon \partial_{\breve{r}} \hat{\theta}\right|_{i \pm} \eta+\left.\frac{1}{2} \epsilon^{2} \partial_{\breve{r} \check{r}} \hat{\theta}\right|_{i \pm} \eta^{2}+\cdots .
\end{aligned}
$$

Replacing the outer expansion into Eq. (118) and grouping terms of the same order leads to

$$
\begin{aligned}
\hat{\theta}= & \hat{\theta}_{0}^{i \pm}+\epsilon\left(\hat{\theta}_{1}^{i \pm}+\left.\partial_{\breve{r}} \hat{\theta}_{0}\right|_{i \pm} \eta\right) \\
& +\epsilon^{2}\left(\hat{\theta}_{2}^{i \pm}+\left.\partial_{\breve{r}} \hat{\theta}_{1}\right|_{i \pm} \eta+\left.\frac{1}{2} \partial_{\breve{r} r} \hat{\theta}_{0}\right|_{i \pm} \eta^{2}\right)+O\left(\epsilon^{3}\right) .
\end{aligned}
$$

Hence, the matching conditions for the temperature are

$$
\begin{gathered}
\lim _{\eta \rightarrow \pm \infty} \theta_{0}=\hat{\theta}_{0}^{i \pm}, \\
\lim _{\eta \rightarrow \pm \infty} \theta_{1}=\hat{\theta}_{1}^{i \pm}+\lim _{\eta \rightarrow \pm \infty}\left(\left.\partial_{\breve{r}} \hat{\theta}_{0}\right|_{i \pm} \eta\right), \\
\lim _{\eta \rightarrow \pm \infty} \theta_{2}=\hat{\theta}_{2}^{i \pm}+\lim _{\eta \rightarrow \pm \infty}\left(\left.\partial_{\breve{r}} \hat{\theta}_{1}\right|_{i \pm} \eta+\left.\frac{1}{2} \partial_{\breve{r} r} \hat{\theta}_{0}\right|_{i \pm} \eta^{2}\right), \\
\lim _{\eta \rightarrow \pm \infty} \partial_{\eta} \theta_{0}=0, \\
\lim _{\eta \rightarrow \pm \infty} \partial_{\eta} \theta_{1}=\left.\partial_{\breve{r}} \hat{\theta}_{0}\right|_{i \pm}, \\
\lim _{\eta \rightarrow \pm \infty} \partial_{\eta} \theta_{2}=\left.\partial_{r} \hat{\theta}_{1}\right|_{i \pm}+\lim _{\eta \rightarrow \pm \infty}\left(\left.\partial_{\breve{r} r} \hat{\theta}_{0}\right|_{i \pm} \eta\right) .
\end{gathered}
$$

\section{A. Phase-field equation at $\boldsymbol{\epsilon}^{\mathbf{0}}$}

Substituting the phase-field inner expansion Eq. (114) into Eq. (112) yields the phase-field equation at the leading order

$$
\partial_{\eta \eta} \psi_{0}+\left(1-\psi_{0}^{2}\right) \psi_{0}=0 .
$$

This equation has for a solution a hyperbolic tangent profile $\psi_{0}=\tanh (\eta / \sqrt{2})$, which constitutes the kernel function of all phenomenological phase-field models.

\section{B. Energy equation at $\epsilon^{0}$}

At the leading order, the energy equation reads

$$
\partial_{\eta}\left(q_{0} \partial_{\eta} \theta_{0}\right)=0 .
$$

Integrating this equation once yields $q_{0} \partial_{\eta} \theta_{0}=A_{0}$, where $A_{0}$ is an integration constant. The matching condition for the temperature at the leading order requires that $\lim _{\eta \rightarrow \pm \infty} \partial_{\eta} \theta_{0}=$ 0 , hence, $A_{0}=0$ and, thus, $\theta_{0}(\eta, s)=\theta_{0}(s)$. This means that the temperature in the inner region can vary along the surface but is constant across the diffuse interface.

\section{Phase-field equation at $\epsilon^{1}$}

The phase-field equation at the first order reads at once

$$
\mathcal{L}\left|\psi_{1}\right\rangle=-\left(\breve{\kappa}+\alpha \breve{u}_{i}-\sqrt{2} \breve{\gamma} \theta_{0}\right)\left|\partial_{\eta} \psi_{0}\right\rangle,
$$

where the bra-ket notation has been introduced to ease the manipulation of internal products. The Hermitian operator is defined as $\mathcal{L}=\partial_{\eta \eta}+\left(1-3 \psi_{0}^{2}\right)$. By differentiating Eq. (126) with respect to $\eta$, we realize that $\mathcal{L}\left|\partial_{\eta} \psi_{0}\right\rangle=0$, which means that the function $\left|\partial_{\eta} \psi_{0}\right\rangle$ is the eigenvector associated to the eigenvalue $\lambda_{0}=0$. This leads to two important conclusions,

$$
\left|\psi_{1}\right\rangle=\left|\partial_{\eta} \psi_{0}\right\rangle
$$


and

$$
\breve{\kappa}+\alpha \breve{u}_{i}-\sqrt{2} \breve{\gamma} \theta_{0}=-\lambda_{0}=0 .
$$

From this result, the inner temperature at the zeroth order reads

$$
\theta_{0}(s)=\frac{\breve{\kappa}+\alpha \breve{u}_{i}}{\breve{\gamma} \sqrt{2}} .
$$

\section{Energy equation at $\epsilon^{1}$}

The energy equation at the first order is given by

$$
\partial_{\eta}\left(q_{0} \partial_{\eta} \theta_{1}\right)-\left(1-(\varpi-1) \theta_{0}\right) \breve{u}_{i} \partial_{\eta}\left(a^{(0)} \partial_{\eta} \phi_{0}+\phi_{0}\right)=0 .
$$

Integrating this equation once leads to

$$
q_{0} \partial_{\eta} \theta_{1}-\left(1-(\varpi-1) \theta_{0}\right) \breve{u}_{i}\left(a^{(0)} \partial_{\eta} \phi_{0}+\phi_{0}\right)=A_{1} .
$$

By having the difference of this expression evaluated at each side of the interface and applying the matching condition for the temperature derivative at the first order, we prove that the Stefan condition is satisfied at the leading order,

$$
\left.q_{0}^{+} \partial_{\breve{r}} \hat{\theta}_{0}\right|^{+}-\left.q_{0}^{-} \partial_{\breve{r}} \hat{\theta}_{0}\right|^{-}=\breve{u}_{i}\left(1-(\varpi-1) \theta_{0}\right) .
$$

Now, we introduce a new dimensionless quantity: the heat flux ratio at the interface $(\chi)$ defined to be

$$
\chi=\frac{\left.q_{0}^{+} \partial_{\breve{r}} \hat{\theta_{0}}\right|^{+}}{\left.q_{0}^{-} \partial_{\breve{r}} \hat{\theta_{0}}\right|^{-}}
$$

and

$$
\Phi(\chi)=\frac{1}{1-\chi} .
$$

These two definitions allow us to to express the heat flux at the vapor side of the interface, in terms of the interface velocity and the function $\Phi$ as

$$
\left.q_{0}^{-} \partial_{\breve{r}} \hat{\theta}_{0}\right|^{-}=-\breve{u}_{i}\left(1-(\varpi-1) \theta_{0}\right) \Phi(\chi) .
$$

This expression is equivalent to the one proposed by Ohno and Matsuura [Eq. (5.1) in Ref. [16]] in their study of solidification of binary alloys. Evaluating Eq. (133) in the bulk vapor yields $\left.q_{0}^{-} \partial_{\breve{r}} \hat{\theta_{0}}\right|^{-}=A_{1}$ and, thus, the evaluation of the integration constant at the liquid side of the interface leads to

$$
A_{1}=-\breve{u}_{i}\left(1-(\varpi-1) \theta_{0}\right) \Phi(\chi) .
$$

By analyzing the behavior of $\Phi$, we realize that this function $\Phi \in[0,1]$ for $\chi \leqslant 0$ (heat is flowing to the interface from both sides). A positive value of $\chi$ indicates that the heat fluxes at both sides of the interface have the same direction, which means that if heat is entering the interface from the liquid side, a certain amount of heat is leaving the interface from the vapor side and vice versa. Although this situation seems very unphysical, it might occur in the presence of kinetic effects. If there is no heat flow to the interface from the vapor side, then $\chi \rightarrow-\infty$ and $\Phi \rightarrow 0$. In real situations, the amount of energy arriving to the interface from the liquid side is always much higher than the one from the vapor side, and, thus, a useful condition in terms of numerics is $|\Phi| \ll 1$. In the case of boiling and for an interface under local thermodynamic equilibrium, no energy can exit the interface through the vapor, thus, $\Phi \in[0,1]$. As pointed out by Ohno and Matsuura, $\Phi$ can be interpreted as a function to improve the convergence of the model. Any value in the interval $[0,1]$ provides physical results, but the convergence of these results towards the sharp-interface solution will vary based on the chosen value of $\Phi$. Integrating the energy equation twice yields

$$
\theta_{1}=A_{2}+\breve{u}_{i}\left(1-(\varpi-1) \theta_{0}\right) \int_{0}^{\eta} p^{(0)} d \eta,
$$

where the function $p^{(0)}$ is defined as

$$
p^{(0)}=\frac{\phi_{0}-\Phi+a^{(0)} \partial_{\eta} \phi_{0}}{q_{0}} .
$$

From this definition, we can express the function $a(\psi)$, present in the antitrapping current, as

$$
a\left(\phi_{0}\right)=\frac{p^{(0)} q_{0}-\phi_{0}+\Phi}{\partial_{\eta} \phi_{0}} .
$$

By evaluating $p^{(0)}$ as $\eta \rightarrow \pm \infty$, we find $p_{ \pm}^{(0)}=\left(\phi_{0}^{ \pm}-\Phi\right) / q_{ \pm}^{(0)}$. Taking the limit of Eq. (139) as $\eta \rightarrow \pm \infty$ and using the matching condition leads to the interface temperature at the first order

$$
\hat{\theta}_{1}^{i \pm}=A_{2}-\breve{u}_{i}\left(1-(\varpi-1) \theta_{0}\right) F^{ \pm},
$$

with

$$
F^{ \pm}=\int_{0}^{ \pm \infty} p_{ \pm}^{(0)}-p^{(0)} d \eta
$$

Having the difference of the interface temperature at both sides of the interface yields

$$
\hat{\theta}_{1}^{i+}-\hat{\theta}_{1}^{i-}=-\breve{u}_{i}\left(1-(\varpi-1) \theta_{0}\right)\left(F^{+}-F^{-}\right) .
$$

We can make this temperature jump at the interface vanish if $F^{+}=F^{-}$. This can be accomplished by defining the following interpolation function for $p^{(0)}$

$$
p^{(0)}=\frac{1}{2}\left(p_{+}^{(0)}+p_{-}^{(0)}+\psi_{0}\left(p_{+}^{(0)}-p_{-}^{(0)}\right)\right) .
$$

In fact any odd function, used to interpolate $p^{(0)}$ across the diffuse interface, eliminates the interface temperature jump.

\section{E. Phase-field equation at $\epsilon^{2}$}

From the phase-field equation at the first order we have that $\breve{\kappa}+\alpha \breve{u}_{i}-\sqrt{2} \breve{\gamma} \theta_{0}=0$, thus, the phase-field equation at the second order reads

$$
\mathcal{L}\left|\psi_{2}\right\rangle=3\left|\psi_{0} \psi_{1}^{2}\right\rangle+\sqrt{2}\left|\theta_{1} \partial_{\eta} \psi_{0}\right\rangle+\frac{1}{2} \breve{\kappa}^{2}\left|\eta \partial_{\eta} \psi_{0}\right\rangle
$$

Since $\mathcal{L}$ is a Hermitian operator, we have that $\left\langle\psi_{i}|\mathcal{L}| \psi_{1}\right\rangle=$ $\left\langle\psi_{1}|\mathcal{L}| \psi_{i}\right\rangle$ and, in particular, if we select $\psi_{i}=\partial_{\eta} \psi_{0}$, we get

$$
\left\langle\partial_{\eta} \psi_{0}|\mathcal{L}| \psi_{1}\right\rangle=\left\langle\psi_{1}|\mathcal{L}| \partial_{\eta} \psi_{0}\right\rangle=0 .
$$

Thus, multiplying Eq. (146) by $\left\langle\partial_{\eta} \psi_{0}\right|$ yields

$\left\langle\partial_{\eta} \psi_{0} \mid \psi_{0} \psi_{1}^{2}\right\rangle+\frac{\sqrt{2}}{3}\left\langle\partial_{\eta} \psi_{0} \mid \theta_{1} \partial_{\eta} \psi_{0}\right\rangle+\frac{\breve{\kappa}^{2}}{6}\left\langle\partial_{\eta} \psi_{0} \mid \eta \partial_{\eta} \psi_{0}\right\rangle=0$.

Since the inner product between odd and even functions is zero, the previous expression reduces to

$$
\left\langle\partial_{\eta} \psi_{0} \mid \theta_{1} \partial_{\eta} \psi_{0}\right\rangle=0 .
$$


By replacing the inner temperature at the first order [Eq. (139) into Eq. (149)], we can express the integration constant $A_{2}$ as

$$
A_{2}=-\breve{u}_{i}\left(1-(\varpi-1) \theta_{0}\right) \frac{K_{0}}{I_{0}},
$$

where the constants $K_{0}$ and $I_{0}$ are defined as

$$
\begin{gathered}
K_{0}=\left\langle\partial_{\eta} \psi_{0} \mid\left(\int_{0}^{\eta} p^{(0)} d \eta\right) \partial_{\eta} \psi_{0}\right\rangle, \\
I_{0}=\left\langle\partial_{\eta} \psi_{0} \mid \partial_{\eta} \psi_{0}\right\rangle .
\end{gathered}
$$

The integral defined in Eq. (152) is identical to that defined in Eq. (76). Substituting Eq. (150) into the interface temperature at the first order [Eq. (142)] gives

$$
\hat{\theta}_{1}^{i \pm}=-\breve{u}_{i}\left(1-(\varpi-1) \theta_{0}\right)\left(F^{ \pm}+\frac{K_{0}}{I_{0}}\right) .
$$

Adding the interface temperature at the zeroth [Eq. (131)] and first [Eq. (153)] orders, we arrive at

$$
\hat{\theta}_{0}^{i \pm}+\epsilon \hat{\theta}_{1}^{i \pm}=\frac{\breve{\kappa}}{\breve{\gamma}} a_{1}+\breve{u}_{i}\left(\frac{\alpha}{\breve{\gamma}} a_{1}-\epsilon\left(1-(\varpi-1) \theta_{0}\right) a_{2}\right),
$$

where $a_{1}=1 / \sqrt{2}$ and $a_{2}=F^{ \pm}+K_{0} / I_{0}$. The dependence of the interface temperature $\hat{\theta}^{i}$ with the interface velocity can be eliminated at the zeroth and first orders if

$$
\alpha=\epsilon \breve{\gamma}\left(1-(\varpi-1) \theta_{0}\right) \frac{a_{2}}{a_{1}} .
$$

After substituting Eq. (155) into the definition of the relaxation time given in Eq. (110), we arrive at

$$
\tau=\frac{W^{3}}{D_{v}} \frac{\gamma I_{0}}{\sigma} \frac{\left(L_{\mathrm{sat}}-\Delta c_{P}^{\mathrm{sat}} \Delta T_{0}\right)}{c_{P v}^{\mathrm{sat}}} \frac{a_{2}}{a_{1}} .
$$

This expression is one of the central results of the thin interface analysis, because it gives us the time scale governing the transformation at mesoscales. The relationship between $\gamma$ and physical properties is given by Eq. (88). By selecting this relaxation time, Eq. (154) becomes

$$
\hat{\theta}_{0}^{i \pm}+\epsilon \hat{\theta}_{1}^{i \pm}=\frac{\breve{\kappa}}{\breve{\gamma}} a_{1}=d_{0} \kappa,
$$

where $\kappa=\breve{\kappa} / l_{c}$ is the dimensional curvature and the thermal capillary length is defined as

$$
d_{0}=\frac{l_{c} a_{1}}{\check{\gamma}}=\frac{\sigma T_{\mathrm{sat}} \rho_{v} c_{P v}\left(1-\rho_{v} / \rho_{l}\right)}{\left(\rho_{v} L_{\mathrm{sat}}\right)^{2}} .
$$

\section{F. Energy equation at $\epsilon^{2}$}

$$
\begin{aligned}
& \partial_{\eta}\left(q^{(0)} \partial_{\eta} \theta_{2}+q_{\psi}^{(0)} \psi_{1} \partial_{\eta} \theta_{1}\right)+\breve{\kappa} q^{(0)} \partial_{\eta} \theta_{1}+\partial_{\eta}\left\{a^{(0)}\left(1-(\varpi-1) \theta_{0}\right) \breve{u}_{i} \partial_{\eta} \phi_{1}+a_{\psi}^{(0)}\left(1-(\varpi-1) \theta_{0}\right) \breve{u}_{i} \psi_{1} \partial_{\eta} \phi_{0}\right. \\
& \left.\quad+a^{(0)}(\varpi-1) \theta_{1} \breve{u}_{i} \partial_{\eta} \phi_{0}\right\}+\breve{u}_{i} \partial_{\eta}\left\{\left(1+(\varpi-1) \phi_{0}\right) \theta_{1}+(\varpi-1)\left(\theta_{0}-1\right) \phi_{1}\right\}+\partial_{s}\left(q^{(0)} \partial_{s} \theta_{0}\right)-a^{(0)}\left(1-(\varpi-1) \theta_{0}\right) \breve{u}_{i} \breve{\kappa} \partial_{\eta} \phi_{0}=0 .
\end{aligned}
$$

Integrating once and taking the limit as $\eta \rightarrow \pm \infty$ leads to

$$
\begin{aligned}
& q_{ \pm}^{(0)}\left(\partial_{\eta} \theta_{2}\right)_{ \pm}+\breve{u}_{i}\left(1+(\varpi-1) \phi_{0}^{ \pm \infty}\right) \theta_{1}^{ \pm \infty}+\int_{0}^{ \pm \infty} \breve{\kappa} q^{(0)} \partial_{\eta} \theta_{1} d \eta \\
& \quad+\int_{0}^{ \pm \infty} \partial_{s}\left(q^{(0)} \partial_{s} \theta_{0}\right) d \eta-\left(1-(\varpi-1) \theta_{0}\right) \breve{u}_{i} \breve{\kappa} \int_{0}^{ \pm \infty} a^{(0)} \partial_{\eta} \phi_{0} d \eta=A_{3} .
\end{aligned}
$$

The matching condition for the temperature derivative at the second order states that $\left(\partial_{\eta} \theta_{2}\right)_{ \pm}=\left.\partial_{\breve{r}} \hat{\theta}_{1}\right|_{i \pm}+\lim _{\eta \rightarrow \pm \infty}\left(\left.\partial_{\breve{r} r} \hat{\theta}_{0}\right|_{i \pm} \eta\right)$ and, thus, we must determine the second derivative of the outer temperature field at the interface $\left.\partial_{\breve{r} r} \hat{\theta}_{0}\right|_{i \pm}$. This is done by extending the outer temperature field [Eq. (113)] into the inner region and evaluating this equation in the limit when $\eta \rightarrow 0$, hence,

$$
\left.q^{ \pm} \partial_{\breve{r r}} \hat{\theta}_{0}\right|_{i \pm}=-\left.\left(q^{ \pm} \breve{\kappa}+\breve{u}_{i}\left(1+(\varpi-1) \hat{\phi}_{0}^{ \pm}\right)\right) \partial_{\breve{r}} \hat{\theta}_{0}\right|_{i \pm}-\left.q^{ \pm} \partial_{s s} \hat{\theta}_{0}\right|_{i \pm} .
$$

Replacing Eqs. (121), (125), (133), (138), and (161) into Eq. (160) leads to

$$
\begin{gathered}
\left.q^{ \pm}\left(\partial_{\breve{r}} \hat{\theta}_{1}\right)\right|_{i \pm}+\breve{u}_{i}\left(1+(\varpi-1) \phi_{0}^{ \pm \infty}\right) \hat{\theta}_{1}^{i \pm}-\lim _{\eta \rightarrow \pm \infty}\left(\left.q^{ \pm} \breve{\kappa} \partial_{\eta} \hat{\theta}_{0}\right|_{i \pm} \eta+q^{ \pm} \partial_{s s} \hat{\theta}_{0} \eta\right) \\
+\int_{0}^{ \pm \infty} \breve{\kappa} \breve{u}_{i}\left(1-(\varpi-1) \theta_{0}\right)\left(\phi_{0}-\Phi\right) d \eta+\int_{0}^{ \pm \infty}\left(q^{(0)} \partial_{s s} \theta_{0}\right) d \eta=A_{3} .
\end{gathered}
$$

Combining Eqs. (133) and (138) and taking the limit as $\eta \rightarrow \pm \infty$ yields

$$
\left.q^{ \pm} \partial_{\breve{r}} \hat{\theta}_{0}\right|_{i \pm}=\left(1-(\varpi-1) \theta_{0}\right) \breve{u}_{i}\left(\phi_{0}^{ \pm}-\Phi\right) .
$$

Replacing Eq. (163) into Eq. (162) and realizing that the derivatives of the temperature along the surface match at all orders $\left(\partial_{s} \theta_{0}=\partial_{s} \hat{\theta}_{0}, \partial_{s s} \theta_{0}=\partial_{s s} \hat{\theta}_{0}, \cdots\right)$, we have

$$
\left.q^{ \pm} \partial_{\breve{r}} \hat{\theta}_{1}\right|_{i \pm}+\breve{u}_{i}\left(1+(\varpi-1) \phi_{0}^{ \pm \infty}\right) \hat{\theta}_{1}^{i \pm}+\breve{\kappa}_{\breve{u}}\left(1-(\varpi-1) \theta_{0}\right) H^{ \pm}+\partial_{s s} \theta_{0} Q^{ \pm}=A_{3},
$$


where the integrals $H^{ \pm}$and $Q^{ \pm}$are defined as

$$
\begin{aligned}
H^{ \pm} & =\int_{0}^{ \pm \infty}\left(\phi_{0}-\phi_{0}^{ \pm \infty}\right) d \eta \\
Q^{ \pm} & =\int_{0}^{ \pm \infty}\left(q_{0}-q_{0}^{ \pm \infty}\right) d \eta
\end{aligned}
$$

By making the difference of Eq. (164) evaluated at the liquid and vapor side of the interface we arrive at

$$
\left.q^{+} \partial_{\eta} \hat{\theta}_{1}\right|_{i+}-\left.q^{-} \partial_{\eta} \hat{\theta}_{1}\right|_{-}=-\breve{u}_{i}(\varpi-1) \hat{\theta}_{1}^{i},
$$

if $H^{+}=H^{-}$and $Q^{+}=Q^{-}$. The first condition eliminates surface stretching [13] and the second one eliminates surface diffusion. By combining Eqs. (134) and (167), it can be proved that the Stefan condition is satisfied at the zeroth and first orders,

$$
\begin{aligned}
& q^{+} \partial_{\eta}\left(\hat{\theta}_{0}+\epsilon \hat{\theta}_{1}\right)_{i+}-q^{-} \partial_{\eta}\left(\hat{\theta}_{0}+\epsilon \hat{\theta}_{1}\right)_{i-} \\
& =\breve{u}_{i}\left(1-(\varpi-1)\left(\hat{\theta}_{0}+\epsilon \hat{\theta}_{1}^{i}\right)\right) .
\end{aligned}
$$

In summary, we have that by choosing the relaxation given by Eq. (156) and satisfying the following set of constraints:

$$
\begin{aligned}
& F^{+}=F^{-}, \\
& H^{+}=H^{-}, \\
& Q^{+}=Q^{-},
\end{aligned}
$$

the present phase-field model converges to the sharp-interface limit as $\sim \epsilon^{2}$. The constraint in Eq. (170) is always satisfied and the other two are fulfilled by choosing interpolation functions, whose derivatives are even functions with respect to the interface position.

\section{ELIMINATION OF THE CURVATURE FROM THE PHASE-FIELD EQUATION}

To understand the details in the elimination of the curvature, let us, first, examine more closely the relaxation time and its impact on each term in the phase-field equation. To do this, we will make use of the relaxation time and the simplified phasefield equation, used in the asymptotic analysis [Eq. (98)] and expressed in terms of the thermal capillary length $d_{0}$ defined in Eq. (158),

$$
\begin{aligned}
\frac{\partial \phi_{l}}{\partial t}= & \frac{1}{\tau}\left(W^{2} \nabla^{2} \phi_{l}+2 \phi_{l}\left(1-\phi_{l}\right)\left(2 \phi_{l}-1\right)\right) \\
& -\frac{1}{\tau}\left(\frac{W}{d_{0}}\right) \frac{a_{1} c_{P v}^{\mathrm{sat}}}{L_{\mathrm{sat}}} 2 \phi_{l}\left(1-\phi_{l}\right) \Delta T
\end{aligned}
$$

and

$$
\tau=\left(\frac{W}{d_{0}}\right) \frac{W^{2}}{D_{v}}\left(1-(\varpi-1) \theta_{0}\right) a_{2} .
$$

The first two terms in right-hand side of Eq. (172) maintain a phase field close to a hyperbolic tangent profile and the third one accounts for the phase change associated to the local superheating. From these two equations, it is clear that the term related to the local superheating is insensitive to the ratio $W / d_{0}$, but the part of the phase-field equation controlling the shape of the profile across the interface is not. When the ratio $W / d_{0}$ is close to 1 , the two main parts composing the phase-field equation have the same order of magnitude, but when $W / d_{0}>$
1, the part controlling the shape of the phase field weakens against the term associated to the phase change and, therefore, the shape of the phase field starts to deviate significantly from the perfect hyperbolic tangent profile. In solidification models, full convergence towards the sharp-interface limit requires $W / d_{0} \sim 1$, which is in line with the current arguments. It is also clear that for $W / d_{0} \ll 1$, the phase field is given by the hyperbolic tangent profile, but this condition requires a very fine grid, since $W$ is usually of the same size as the grid spacing $\Delta x$. The term controlling the shape of the phase field also carries implicitly (within the Laplacian) the curvature of the interface, and, therefore, also controls the local superheating associated to curvature effects. To obtain accurate values for the curvature-related superheating, it also necessary to have $W / d_{0} \sim 1$ and, therefore, simulations at length scales where $W / d_{0} \gg 1$ are not physically valid. A solution to this problem is the elimination of the curvature from the Laplacian and, thus, the superheating associated to curvature effects also vanishes. In addition, and since, at the sharp-interface limit $\left(W / d_{0}<1\right)$, the phase field is given by the hyperbolic tangent profile, a split of the phase-field equation into two equations would allow us to enforce a hyperbolic tangent profile independently from the term associated to the phase change. Hence, the new equations replacing Eq. (172) are

$$
\begin{gathered}
\tilde{\mu}_{1}=\frac{4 \sigma}{W I_{0}} \frac{W}{d_{0}} \frac{a_{1} c_{P v}^{\mathrm{sat}}}{L_{\mathrm{sat}}} 2 \phi_{l}\left(1-\phi_{l}\right) \Delta T, \\
\frac{\partial \phi_{l}}{\partial t}=-M \tilde{\mu}_{1}, \quad \text { with } M=\frac{W I_{0}}{4 \sigma \tau}, \\
\frac{\partial \phi_{l}}{\partial t^{*}}=W^{2}\left(\nabla^{2} \phi_{l}-\kappa|\nabla \psi|\right)+2 \phi_{l}\left(1-\phi_{l}\right)\left(2 \phi_{l}-1\right) .
\end{gathered}
$$

The third equation is only used to enforce the hyperbolic tangent profile after the phase field has been modified due to phase change. A hyperbolic tangent profile will be achieved when $\partial \phi_{l} / \partial t^{*}=0$ and, thus, the time $t^{*}$ does not correspond to a physical time and must be interpreted as an auxiliary variable to drive $\phi_{l}$ to the desired profile. This equation represents a nonconservative resharpening step, which does not keep the integral of $\phi_{l}$ constant during the resharpening procedure. This is an undesired property in a resharpening equation, which can be easily solved by expressing the equation in a conservative way. Considering that $\phi_{l}$ is truly given by the hyperbolic tangent $\phi_{l}=[1+\tanh (r / W \sqrt{2})] / 2$, then a conservative version of Eq. (176) reads

$$
\frac{\partial \phi_{l}}{\partial t^{*}}=\nabla \cdot\left(W^{2} \nabla \phi_{l}-W \sqrt{2} \phi_{l}\left(1-\phi_{l}\right) \frac{\nabla \phi_{l}}{\left|\nabla \phi_{l}\right|}\right) .
$$

This equation is completely equivalent to the equation proposed by Olsson and Kreiss [35] as a resharpening step. It is important to note that the split of the phase-field equation and the elimination of the curvature are only possible because, first, the surface energy is isotropic and, second, when studying boiling at scales ranging from micrometers to millimeters, curvature effects are negligible. For nanoscale systems, the conservative re-sharpening equation is still valid and useful to maintain the shape of the phase field during strongly advecting flows. Nonetheless, the original phase-field equation is required to account properly for curvature effects in the interface temperature. Furthermore, the definition for the 
relaxation time is unrestrictive of the length scale of the system under study.

\section{SOLUTION OF THE MODEL}

Before presenting the algorithm for solving the present model, a few remarks are necessary in order to clarify some details in the field equations. When deriving Eq. (43), we required that $\nabla \cdot \overline{\mathbf{u}}=\Delta \overline{\mathbf{u}} \cdot \nabla \phi_{l}$, which corresponds to an even distribution across the diffuse interface if the phase field is given by the hyperbolic tangent profile. From Eq. (23) we have that, if $\nabla \cdot \overline{\mathbf{u}}$ is an even function, the evaporation $\ddot{\Gamma}_{l}$ must also be evenly distributed across the interface, but if we calculate the evaporation rate as

$$
\ddot{\Gamma}_{l}=\rho_{v} M \tilde{\mu}_{1},
$$

the evaporation rate will not be an even function for nonisothermal systems, due to the temperature variation across the interface. An evenly distributed evaporation rate is fundamental for the validity of Eqs. (91), (93), and (94), and, therefore, a special algorithm is required to change the asymmetric evaporation rate Eq. (178) into an even distribution, without changing the amount of evaporated liquid. This can be accomplished by the introduction of an auxiliary phase field $\phi_{l}^{*}$ and the solution of a conservative resharpening equation. Hence, the algorithm to solve the full model in each time step, including a symmetric evaporation rate, is as follows:

(1) Calculate the transformation potential $\tilde{\mu}_{1}$ from Eq. (174).

(2) Solve the pseudo phase field (only needed for the calculation of the evaporation rate)

$$
\phi_{l}^{*}=\phi_{l}^{(0)}-\Delta t M \tilde{\mu}_{1},
$$

where $\phi_{l}^{(0)}$ is the phase field in the previous physical time step. The relaxation time $\tau$ is determined by Eq. (173) and the term $(\varpi-1) \theta_{0}$ can be safely dropped for system temperatures lower than the critical one.

(3) Solve the resharpening equation for the pseudo phase field

$$
\frac{\partial \phi_{l}^{*}}{\partial t^{*}}=\nabla \cdot\left(W^{2} \nabla \phi_{l}^{*}-W \sqrt{2} \phi_{l}^{*}\left(1-\phi_{l}^{*}\right) \frac{\nabla \phi_{l}}{\left|\nabla \phi_{l}\right|}\right) .
$$

(4) Calculate the symmetric evaporation rate as

$$
\ddot{\Gamma}_{l}=-\rho_{v}\left(\frac{\phi_{l}^{*}-\phi_{l}^{(0)}}{\Delta t}\right) .
$$

(5) Solve the mass Eq. (91) and momentum Eq. (92) equations (by any coupling technique) with the symmetric evaporation rate. The surface tension force is calculated from $\mathbf{f}_{l v}^{\sigma}=\hat{\mu} \nabla \phi_{l}$, where $\hat{\mu}$ is defined in Eq. (77).

(6) Solve the phase-field equation with convection and phase change. The term $\psi \nabla \cdot \overline{\mathbf{u}}$ is an odd function and, therefore, it can only change the shape of the phase-field profile, not the interface position. Hence, after a conservative resharpening step, any change introduced by this term to the phase-field profile will vanish. For this reason, the term $\psi \nabla \cdot \overline{\mathbf{u}}$ can be dropped from the phase-field equation, when
TABLE I. Liquid properties.

\begin{tabular}{lcccccc}
\hline \hline Fluid & $\begin{array}{c}P \\
(\text { bar })\end{array}$ & $\begin{array}{c}\rho_{l} \\
\left(\frac{\mathrm{kg}}{\mathrm{m}^{3}}\right)\end{array}$ & $\begin{array}{c}c_{P l} \\
\left(\frac{\mathrm{J}}{\mathrm{kg} \cdot \mathrm{K}}\right)\end{array}$ & $\begin{array}{c}k_{l} \\
\left(\frac{\mathrm{W}}{\mathrm{m} \cdot \mathrm{K}}\right)\end{array}$ & $\begin{array}{c}\mu_{l} \\
\left(\frac{\mathrm{N} \cdot \mathrm{s}}{\mathrm{m}^{2}}\right)\end{array}$ & $\begin{array}{c}\sigma \\
\left(\frac{\mathrm{J}}{\mathrm{m}^{2}}\right)\end{array}$ \\
\hline Water $^{\mathrm{a}}$ & 1 & 958.4 & 4215.9 & 0.677 & $2.8 \times 10^{-4}$ & 0.0589 \\
Water $^{\mathrm{a}}$ & 0.39 & 974.5 & 4193.1 & 0.663 & $3.7 \times 10^{-4}$ & 0.0635 \\
Ethanol $^{\mathrm{b}}$ & 1 & 758.3 & 3005.0 & 0.169 & $4.5 \times 10^{-4}$ & 0.0175 \\
\hline \hline
\end{tabular}

${ }^{a}$ ASME steam table.

${ }^{\mathrm{b}}$ Thermal-Fluids Central. Available online at https://www. thermalfluidscentral.org/encyclopedia/index.php.

introducing a conservative resharpening equation,

$$
\frac{\partial \phi_{l}}{\partial t}+\nabla \cdot\left(\phi_{l} \overline{\mathbf{u}}\right)=-\frac{\ddot{\Gamma}_{l}}{\rho_{l}} .
$$

(7) Solve the resharpening equation for the phase field.

(8) Solve the energy equation Eq. (93).

For small systems, where curvature effects are important, the only difference in the solution algorithm would be in the first step, where the original transformation potential, defined in Eq. (84), should be used instead of $\tilde{\mu}_{1}$.

\section{RESULTS}

In this section, phase-field simulations are compared to onedimensional analytical solutions and experimental results of bubble growth in superheated liquids. The physical properties for the studied fluids (water and ethanol) are listed in Tables I and II.

\section{A. Numerical tests}

To assess the phase-field model, several physical situations were addressed, considering different degrees of superheating and saturation pressures. All simulations were performed in one-dimensional setups in Cartesian or spherical coordinates, with water at 1 bar as the test fluid. The backward Euler scheme was used for time discretization and the finite volume method for the spatial discretization of all the conservation equations, considering a staggered grid for the velocity field. The SIMPLE algorithm was used to enforce mass conservation. In all the simulations, $W$ was related to the grid spacing through $W=1.3 \Delta x$ and the value for $\Phi$ was equal to zero unless stated otherwise.

\section{Confined vapor $A$}

In the first case under study, the heat is transported from

\begin{tabular}{|c|c|c|c|c|c|c|}
\hline Fluid & $\begin{array}{c}P \\
\text { (bar) }\end{array}$ & $\begin{array}{c}\rho_{v} \\
\left(\frac{\mathrm{kg}}{\mathrm{m}^{3}}\right)\end{array}$ & $\begin{array}{c}c_{P v} \\
\left(\frac{\mathrm{J}}{\mathrm{kg} \cdot \mathrm{K}}\right)\end{array}$ & $\begin{array}{c}k_{v} \\
\left(\frac{\mathrm{W}}{\mathrm{m} \cdot \mathrm{K}}\right)\end{array}$ & $\begin{array}{c}\mu_{v} \\
\left(\frac{\mathrm{N} \cdot \mathrm{s}}{\mathrm{m}^{2}}\right)\end{array}$ & $\begin{array}{c}L \\
\left(\frac{\mathrm{J}}{\mathrm{kg}}\right)\end{array}$ \\
\hline Water & 1 & 0.59 & 2026.1 & 0.025 & $1.2 \times 10^{-5}$ & 2257826 \\
\hline Water & 0.39 & 0.24 & 1949.0 & 0.022 & $1.1 \times 10^{-5}$ & 2320657 \\
\hline Ethanol & 1 & 1.36 & 1603.0 & 0.020 & $1.0 \times 10^{-5}$ & 962890 \\
\hline
\end{tabular}
the wall to the interface through the confined vapor phase (see Fig. 6). The wall superheating is set to $\Delta T_{w}=10 \mathrm{~K}$ and the system size is $1 \mathrm{~mm}$. The initial temperature distribution was

TABLE II. Vapor properties. 


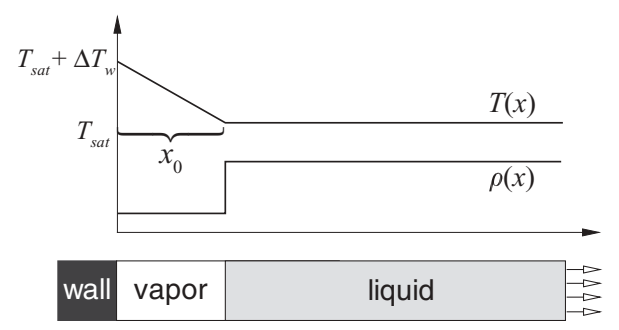

FIG. 6. Schematic for the test case with heat diffusing through the confined vapor phase.

obtained from the analytical solution for an interface position equal to $x_{0}=0.1 \mathrm{~mm}$. This is the well-known Stefan problem, where the advection of the liquid phase is irrelevant.

The analytical solution to this problem is obtained by using the similarity variable $\xi(x, t)=x \beta / x_{i}(t)$ and assuming the interface at the saturation temperature. Thus, the temperature distribution, interface position, and the constant $\beta$ are given by

$$
\begin{gathered}
T(x, t)= \begin{cases}T_{\mathrm{wall}}-\Delta T_{w} \frac{\operatorname{erf}(\xi)}{\operatorname{erf}(\beta)} & \text { if } x \leqslant x_{i} \\
T_{\mathrm{sat}} & \text { if } x>x_{i},\end{cases} \\
x_{i}(t)=2 \beta \sqrt{D_{v} t}, \\
\beta e^{\beta^{2}} \operatorname{erf}(\beta)=\frac{c_{P v}^{\text {sat }} \Delta T_{w}}{\sqrt{\pi} L_{\mathrm{sat}}} .
\end{gathered}
$$

This test case constitutes the best way to evaluate the influence of $\Phi$ on the convergence of the current model towards the sharp-interface limit. As stated in asymptotic analysis, this parameter must be between 0 and 1 for boiling systems, where extremes $(\Phi=0$ or $\Phi=1)$ represent a physical situation where the heat is flowing to the interface only from the liquid or vapor side, respectively. For the present case, the heat is flowing only from the vapor side and, therefore, a value of $\Phi$ equal to 1 should produce the best results. This was confirmed by running simulations of the two extreme cases, with four levels of mesh refinement. In Fig. 7, the interface is plotted against time, displaying a mild dependence on $\Phi$. To further

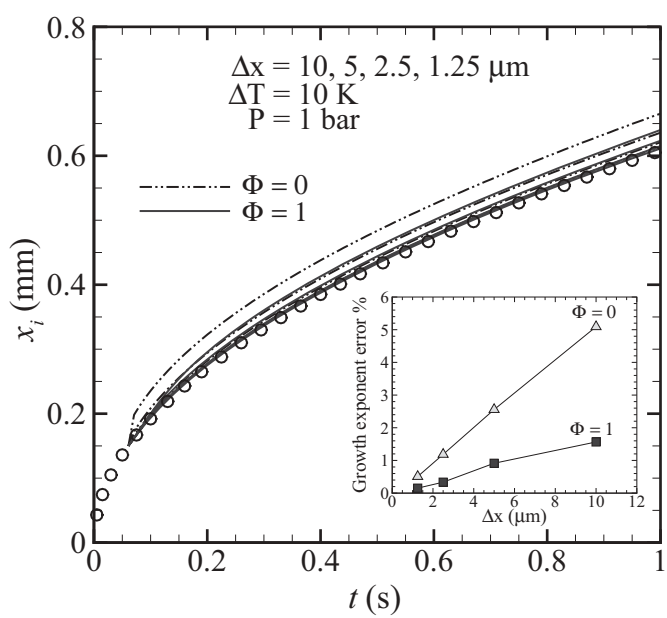

FIG. 7. Interface position for the case Confined vapor A. Symbols represent the analytical solution. Phase-field simulations are denoted by lines. examine the convergence of the model on $\Phi$, I have obtained the growth exponent from the numerical results and compared it with the one from the analytic solution (which is equal to $1 / 2$ ). To avoid including numerical results influenced by the initial transient induced by the sharp temperature distribution, a power-law curve fitted from 0.9 to $1 \mathrm{~s}$, was used to obtain the growth exponent. The dependence of the relative error with $\Phi$ and the grid spacing $\Delta x$ is presented in the inset of Fig. 7. For more realistic boiling situations, the heat flux to the interface at the vapor side is always much smaller than the one at the liquid side and, therefore, $\Phi \sim 0$. In fact, all the rest of the simulations carried out in this work consider $\Phi=0$.

\section{Confined vapor $B$}

A second test case with a confined vapor phase is used to assess the phase-field model when the interface velocity is controlled by a dynamic thin thermal boundary layer in the liquid phase (see Fig. 1). This problem is particularly challenging because involves phase change, and the advection of the phase field and thermal boundary layer, due to the pushing effect of the expansion of the liquid during its evaporation. As in the previous case, this problem also finds a simple analytical solution in terms of a similarity variable $\xi(x, t)=\left(x-x_{i}(t)\right) / \sqrt{2 D_{l} t}$, leading to the following expressions for the temperature distribution, interface position, and the constant $\beta$,

$$
\begin{gathered}
T(x, t)=T_{\text {sat }}+\Delta T_{\infty}\left(1-\frac{\operatorname{erfc}\left(\frac{\xi}{\sqrt{2}}+\beta\right)}{\operatorname{erfc}(\beta)}\right), \\
x_{i}(t)=\frac{\rho_{l}}{\rho_{v}} 2 \beta \sqrt{D_{l} t}, \\
\beta e^{\beta^{2}} \operatorname{erfc}(\beta)=\frac{c_{P l}^{\text {sat }} \Delta T_{\infty}}{\sqrt{\pi} L_{\text {sat }}} .
\end{gathered}
$$

Note that the density ratio $\rho_{l} / \rho_{v}$ has been introduced in the equation for the interface position, which accounts for the volume change during the liquid expansion. In contrast to the previous case, all the properties are now referred to as the liquid phase. The simulated system size was $10 \mathrm{~mm}$ and the initial interface position was obtained from the analytical solution for a time equal to $0.1 \mathrm{~s}$. Since interface velocity is high for this case, an appropriate initial temperature distribution inside the diffuse interface acquires special relevance. To find a smoothly varying temperature distribution across the diffuse interface (see Fig. 5), we can make use of the temperature distribution at the zeroth and first orders, derived from the asymptotic analysis. The dimensionless temperature distribution at the zeroth and first order can be obtained by combining Eqs. (131), (139), (150), and (C1), which yields

$$
\theta_{0}+\epsilon \theta_{1}=\breve{u}_{i} \epsilon p_{+}^{(0)}\left\{\frac{\sqrt{2}}{2} \ln \left(\frac{2}{\psi+1}\right)+\eta\right\} .
$$

This equation approaches asymptotically a linear distribution in the bulk liquid and goes to zero in the bulk vapor. For converged results, this expression matches the analytical solution at the matching boundaries between the inner and outer regions (see Fig. 5). Hence, by substracting $\breve{u}_{i} \in p_{+}^{(0)} H(\eta) \eta$, with $H(\eta)$ the Heaviside function, we obtain the departure of the temperature distribution from the analytical one, at the 


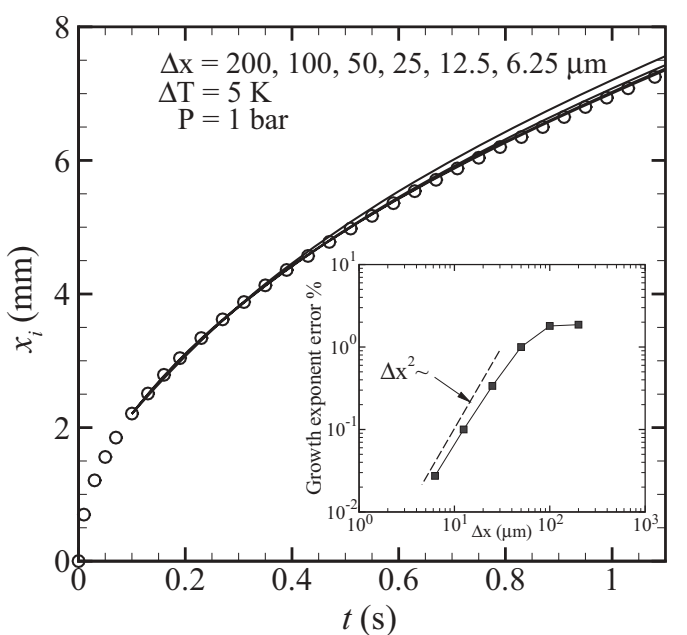

FIG. 8. Interface position for the case Confined vapor B. Symbols represent the analytical solution. Phase-field simulations are denoted by lines.

zeroth and first orders. In dimensional form, the temperature departure from the analytical solution reads

$$
\Delta T(x)=\frac{L_{\mathrm{sat}}}{c_{P v}^{\mathrm{sat}}} \frac{u_{i}}{D_{v}} p_{+}^{(0)}\left\{\frac{\sqrt{2}}{2} \ln \left(\frac{2}{\psi+1}\right)+x(1-H(x))\right\} .
$$

By adding this expression to the analytical temperature distribution, we obtain a temperature profile which approaches that of the phase-field model up to the first-order corrections. To initialize the temperature distribution in the simulations, the interface velocity was obtained from the analytical expression for the interface position. Figure 8 presents the predicted interface position along with the analytical solution for six levels of mesh refinement. Although coarse grids produced good results, finer grids were required to analyze the convergence of the model towards the sharp-interface solution. The convergence study was done using the relative error in the growth exponent for the last part of the simulations.

The inset in Fig. 8 shows that, for very fine grids, the phasefield model approaches the sharp-interface limit as $\sim \Delta x^{2}$. It is interesting to see that the error does not increase in a $\Delta x^{2}$ fashion for coarse grids, which implies that the solution does not deteriorate as fast as those in models with a purely quadratic convergence rate. The temperature distribution for the six levels of mesh refinement is presented in Fig. 9, where the inset shows that fine grids are truly converging to the sharp-interface limit. Analyzing in detail the results from Fig. 9, it can be seen that, within the diffuse interface, the temperature has an unusual distribution. This is related to higher-order terms in the temperature distribution (see the energy equation at $\epsilon^{2}$ in the asymptotic analysis), which depend on the interface velocity, property ratios, and interface thickness. If we were to use this model at nanoscales, the interface thickness would be so small (few angstroms) that any effect arising from higher-order corrections would be dwarfed by the leading terms. It is important to mention that within the diffuse interface, the heat flux does not follow exactly Fourier's law due to the phenomenological antitrapping current, required to balance

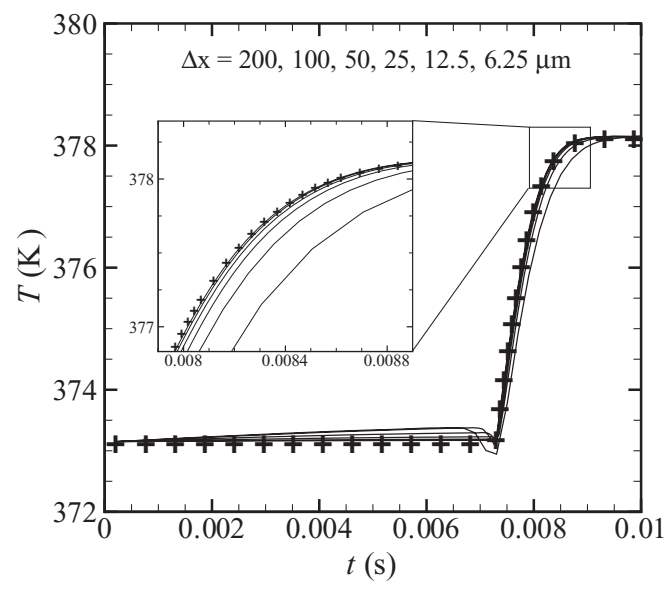

FIG. 9. Temperature distribution at $1 \mathrm{~s}$ for the case Confined vapor B. Symbols represent the analytical solution. Phase-field simulations are denoted by lines.

the spurious effect arising from the steep variation of the thermal conductivity across the diffuse interface. Since there is no way to experimentally investigate how the thermal energy is transported inside a real interface, the physical validity of the antitrapping current can neither be proved nor discarded. Thus, in the context of phase-field modeling, it must regarded simply as a tool to improve the convergence towards the sharp-interface limit, making possible simulations with coarser grids. McFadden et al. [36] also added a nonconventional current to the classical heat flux in their solidification model with unequal thermal conductivities. They also brought up the question about the physical validity of these nonconventional terms, but they stated that, independently of their physical validity, they facilitate the numerical solution of the model, which is in the same line of reasoning discussed in this article. Despite the fact that, within the diffuse interface, the temperature distribution appears to display a departure from what might be considered "normal," the accurate prediction of interface position guarantees the correct evaluation of the evaporation rate, which is the most relevant aspect of the boiling process.

\section{Confined liquid}

Another interesting test case is obtained by exchanging the position of the liquid and vapor phases in the first test case (confined vapor $A$ ), while keeping all the parameters unaltered and setting the interface position at $x_{0}=0.5 \mathrm{~mm}$. In this new situation, which resembles an evaporating thin liquid film, the velocity in the liquid phase is zero and, therefore, the pushing effect of the liquid expansion only affects the vapor phase, which is considered to be at a constant temperature and equal to the saturation one. In the first test case, the Stefan solution requires a step function for the initial temperature distribution, which introduces a singularity in the temperature profile right at the interface. In contrast, in this third case, the initial temperature distribution is linear across the liquid phase. This particular fact impedes the use of the Stefan solution on a time reversal and the change of properties from vapor to liquid in all the equations. In spite of this, the use of the similarity variable $\xi(x, t)=x \beta / x_{i}(t)$ leads to a new analytical solution 


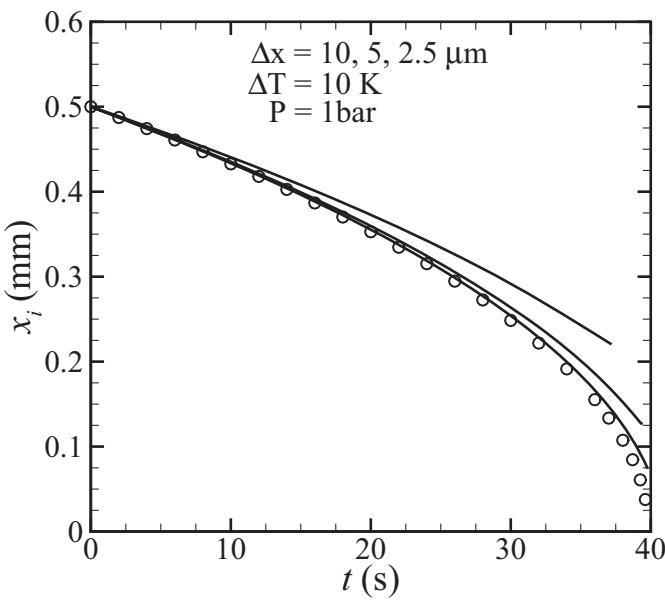

FIG. 10. Interface position for the case test Confined liquid. Symbols represent the analytical solution. Phase-field simulations are denoted by lines.

in terms of the imaginary error function erfi $(x)=-i \operatorname{erf}(i x)$, given by

$$
\begin{gathered}
T(x, t)= \begin{cases}T_{\mathrm{wall}}-\Delta T_{w} \frac{\operatorname{erfi}(\xi)}{\operatorname{erf}(\beta)} & \text { if } x \leqslant x_{i} \\
T_{\mathrm{sat}} & \text { if } x>x_{i},\end{cases} \\
x_{i}(t)=2 \beta \sqrt{D_{l}\left(t_{0}-t\right)}, \\
\beta e^{-\beta^{2}} \operatorname{erfi}(\beta)=\frac{c_{P l} \Delta T_{w}}{\sqrt{\pi} L_{\mathrm{sat}}} .
\end{gathered}
$$

The characteristic time $t_{0}$ can be obtained directly from Eq. (192) for a given position of the interface at $(t=0)$, hence, $t_{0}=x_{0}^{2} /\left(4 D_{l} \beta^{2}\right)$. The interface position is shown in Fig. 10. For this case, only three levels of mesh refinement were used. The results show an steady convergence towards the sharp-interface limit.

\section{Confined vapor in spherical coordinates}

This is the most relevant test case since it is the closest to a real boiling situation. The schematic for the physical situation addressed in this case is presented in Fig. 11. As in the second test case, the interface velocity is also controlled by a thin thermal boundary layer, but the main difference lies in the introduction of the curvature in the analytical solution. Scriven [37] derived the analytical solution for this problem based on

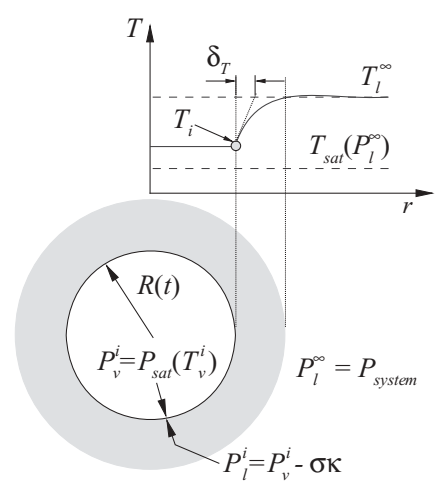

FIG. 11. Schematic for the confined vapor in spherical coordinates. one particular assumption, that the interface temperature does not depend on the interface curvature and is set to the saturation point. Plesset and Zwick [38] also derived an analytical expression for the bubble size and temperature distribution without assuming the interface at the saturation temperature. Instead, they used perturbation theory to find the solution in terms of an infinite series, where higher-order terms represent successive corrections to the leading term. Nonetheless, these authors only presented the first two terms of the entire solution, due to the increasing mathematical complexity of higher-order terms. Today, no analytical solution exists for the problem of bubble growth, including curvature effects on the interface temperature, but the Scriven results represent a complete solution to the asymptotic case, where the interface is thought to be independent of the local curvature. The elimination of the curvature from the transformation potential in the current model allows for a direct comparison with Scriven's solution. However, it is important to remark that the elimination of the curvature is only due to computer limitations. Leaving the curvature in the transformation potential leads to numerical solution of the complete problem of bubble growth in a superheated liquid, but the solution would be possible only for very small systems. Using the similarity variable expressed in terms of the bubble radius $\xi(r, t)=r \beta / R(t)$ and considering all the physical properties evaluated at the saturation temperature, Scriven's solution is given by

$$
\begin{gathered}
R(t)=2 \beta \sqrt{D_{l} t} \\
T(r, t)=T_{\infty}-A \int_{1-\frac{R(t)}{r}}^{1} e^{-\beta^{2}\left((1-u)^{-2}-2 \epsilon u-1\right)} d u \\
2 \beta^{2} \int_{0}^{1} e^{-\beta^{2}\left((1-u)^{-2}-2 \epsilon u-1\right)} d u=\frac{\rho_{l}}{\rho_{v}} \frac{c_{P l} \Delta T_{\infty}}{L},
\end{gathered}
$$

where $\Delta T_{\infty}=T_{\infty}-T_{\text {sat }}$. In his original article, Scriven chose the liquid far from the interface as the reference state, and, thus, all the physical properties were evaluated at $T_{\infty}$. For constant properties (which requires a linearization of the latent heat), the selection of the reference state at $T_{\text {sat }}$ or $T_{\infty}$ changes slightly the energy balance at the interface, but at temperatures away from the critical temperature, the choice of the reference state is irrelevant. For the case of a confined vapor phase, the energy balance at a sharp interface reads

$$
\rho_{v} L(T) \dot{R}=\left.k_{l} \frac{\partial T}{\partial r}\right|_{i},
$$

where the latent heat $L(T)=h_{v}(T)-h_{l}(T)$ is defined as the difference between the vapor and liquid enthalpies at a given temperature $T$. Using a linear expansion of the enthalpies around a reference temperature $T_{a}$ yields $L(T)=$ $L\left(T_{a}\right)+\left(c_{P v}^{a}-c_{P l}^{a}\right)\left(T-T_{a}\right)$. Replacing this expression into Eq. (197) leads to

$$
\rho_{v} \dot{R}\left(L_{a}+\left(c_{P v}^{a}-c_{P l}^{a}\right)\left(T_{i}-T_{a}\right)\right)=\left.k_{l}^{a} \frac{\partial T}{\partial r}\right|_{i},
$$

where $T_{i}$ is the interface temperature. In the present work, I have chosen the saturation temperature as the reference state ( $\left.T_{a}=T_{\text {sat }}\right)$, which appears to be a more suitable selection since the evaporation phenomena is taking place at the interface and not away from it. As in Scriven's work, I have also assumed 


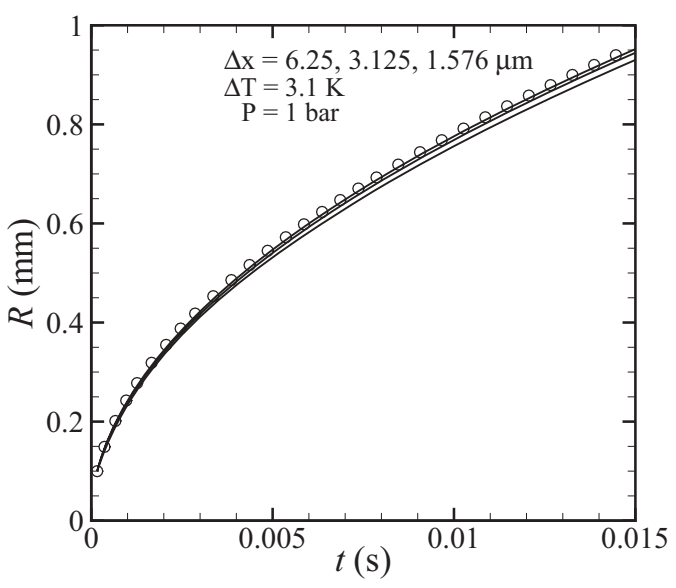

FIG. 12. Comparison between phase-field prediction of bubble size with the analytical solution derived by Scriven (represented by symbols). Phase-field simulations are denoted by lines.

the interface to be at the saturation temperature and, therefore, the term related to the temperature difference vanishes, which is nonzero when selecting $T_{a}=T_{\infty}$. One-dimensional spherically symmetric phase-field simulations were carried out for a system size of $1.5 \mathrm{~mm}$ and a liquid superheating equal to $\Delta T_{\infty}=3.1 \mathrm{~K}$. The initial condition for the temperature distribution was obtained from Scriven's solution for a bubble size of $R_{0}=0.1 \mathrm{~mm}$. A comparison between the predicted bubble size and Scriven's analytical solution is presented in Fig. 12. A direct comparison between phase-field simulations and experimental results will be presented in the next section, where the initial bubble size and thermal boundary layer thickness were directly obtained from the experiments.

Three mesh sizes were used to analyze the convergence of the model $\Delta x=6.25,3.125$, and $1.576 \mu \mathrm{m}$. Since the bubble size increases almost 10-fold during the simulation, converged results required a fine grid with large number of points, $n x=$ 960. The large number of grid points can be explained in terms of the initial thermal boundary layer thickness. By combining Scriven's solution with the Stefan condition (energy balance at the sharp interface), it is possible to relate the thermal boundary layer thickness, which is defined as $\delta_{T}=\left(T_{\infty}-T_{i}\right) /(\partial T / \partial r)_{i}$, to the bubble size by

$$
\delta_{T}=\frac{\rho_{l}}{\rho_{v}} \frac{c_{P l}^{\mathrm{sat}} \Delta T_{\infty}}{2 \beta^{2} L_{\mathrm{sat}}} R(t),
$$

which indicates a constant ratio of the thermal boundary layer thickness to the bubble size during bubble growth.

With this expression, the extent of the thermal boundary layer can be estimated directly from the bubble size. For a bubble size of $0.1 \mathrm{~mm}$ and superheating equal to $\Delta T_{\infty}=3.1 \mathrm{~K}$, the thermal boundary layer thickness is $\delta_{T}=5.3 \mu \mathrm{m}$ and, therefore, a grid spacing of $\Delta x=1.57 \mu \mathrm{m}$ is just enough to resolve the temperature distribution around the bubble. An interesting observation from Eq. (199) is that for this set of parameters and physical properties, the bubble radius is almost 20 times larger than the thermal boundary layer thickness. Hence, a large number of grid points are required to resolve simultaneously the bubble size and the thermal boundary layer.

\section{B. Validation against experimental results}

One of the main problems in comparing numerical simulations with experiments lies in the selection of the appropriate initial conditions. For instance, an experimentally measured temperature distribution around a freshly nucleated bubble could be set as initial condition, but this type of measurements appears to be rather difficult and perhaps not justified. An additional degree of uncertainty in the initial conditions, is introduced by the nucleation procedure, which varies from author to author. For example, Dergarabedian [39] used small particles of chalk or sand to nucleate bubbles with different level of superheating, whereas Lien [40] used an electric discharge between two thin electrode wires, creating a sudden formation of a small amount of electrolytic gases, serving as an stable nucleus for a single vapor bubble. Although the nucleation procedure differed between these two authors, Lien showed that he could closely reproduced Dergarabedian's results, which implies a bubble growth independence from the nucleation procedure. This particular fact could be used to find a way to infer a suitable initial condition for the simulations. Most of the experiments carried out by Lien and Dergarabedian are in the diffusion-controlled regime, and, therefore, it should be possible, in principle, to modify Scriven's solution to include an arbitrary initial bubble size $R_{0}$, which would account for the nucleation process. This could be done by rewriting Eq. (194) as

$$
R(t)^{2}=4 \beta^{2} D_{l} t
$$

and then substracting from it the same equation evaluated at an arbitrary time $t_{0}$, leading to

$$
R(t)=\sqrt{R_{0}^{2}+4 \beta^{2} D_{l}\left(t-t_{0}\right)} .
$$

By expressing the bubble radius in terms of an arbitrary initial time $t_{0}$, we are moving Scriven's solution to the left or to the right to better capture interface velocity, but to fit the experimental bubble size, it is necessary to further modify this equation as

$$
R^{*}(t)=R(t)-R_{0} .
$$

This allows us to have a bubble size equal to $R^{*}=0$ at $t=t_{0}$. All the experimental results analyzed in this work could be accurately fitted by Eq. (202). Nonetheless, only Eq. (201) satisfies the solution for the sharp-interface model; hence, to obtain the temperature distribution at any time, we must substitute Eq. (201) into Eq. (195). Since the interface velocity in sharp-interface models is controlled by the temperature gradient at the liquid-vapor interface (and not by the bubble size), the main idea of the fitting procedure is to find a bubble size, from the Scriven's solution, that produces an interface temperature distribution that closely matches the experimental interface velocity. Figure 13 shows the fit of one of the experiments carried out by Lien at reduced pressure. The constants $R_{0}$ and $t_{0}$ are obtained from the experiments through the fit and the constant $\beta$ is obtained from Eq. (196). Several conditions and substances were simulated in order to validate the phase-field model presented in this work. The most challenging conditions for simulations are those of water at reduced pressure, where the density ratio reaches 4000 . This creates a very thin thermal boundary layer and high 


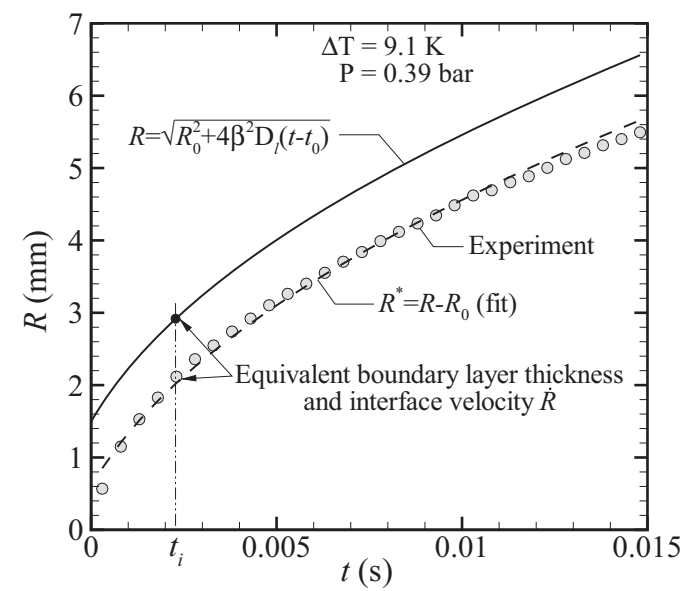

FIG. 13. Fit of the experimental results. This fitting procedure is required to obtain the appropiate initial temperature distribution for the phase-field simulations.

interface velocities. Figure 14 shows the phase-field prediction of bubble size for water at $P=1 \mathrm{bar}$ and $\Delta T_{\infty}=9.1 \mathrm{~K}$, which correspond to the experimental conditions set by Lien for bubble 8 in his Ph.D. thesis [40]. An initial bubble size of $R\left(t_{i}\right)=2.1 \mathrm{~mm}$ was directly obtained from the experiment at a time equal to $t_{i}=2.3 \mathrm{~ms}$ and system size was $9 \mathrm{~mm}$. Due to the thin thermal boundary layer, a large number of grid points was required for converged results $(n x=3000)$. Dergarabedian estimated a total uncertainty of $10 \%$ in the bubble size, due to uncertainties in the temperature, system pressure, and bubble size measurements. This number seems to be conservative and reasonable, thus two lines accounting for this uncertainty were included in all the comparisons with experimental results.

A second comparison was carried out for water at $P=1$ bar. Experiments from Dergarabedian as well as Florschuetz et al. [41] at slightly different superheatings were combined into a single plot, providing a good opportunity for evaluating the uncertainty in the bubble size measurements. Figure 15 presents phase-field simulations along with experimental results from different sources. The dispersion of the experimental

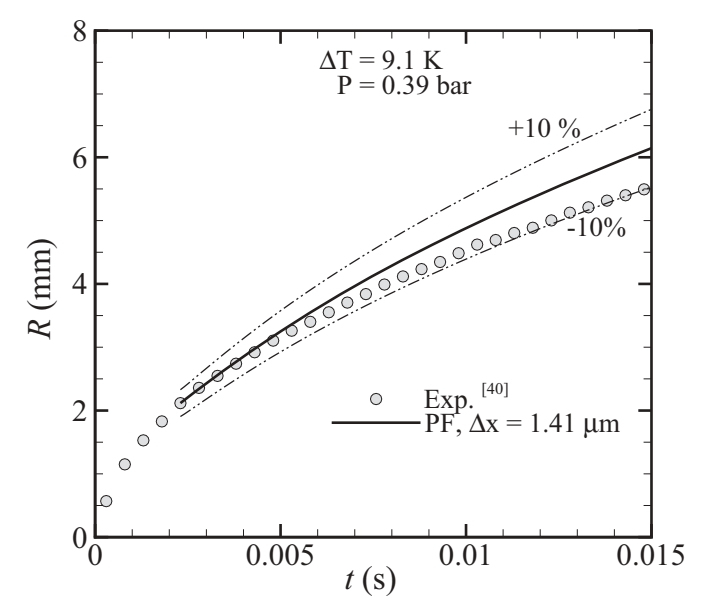

FIG. 14. Comparison between phase-field simulations and experimental results [40] for bubble growth of water at reduced pressure. Experiments are diplayed in symbols and phase-field simulations are shown in continuous lines.

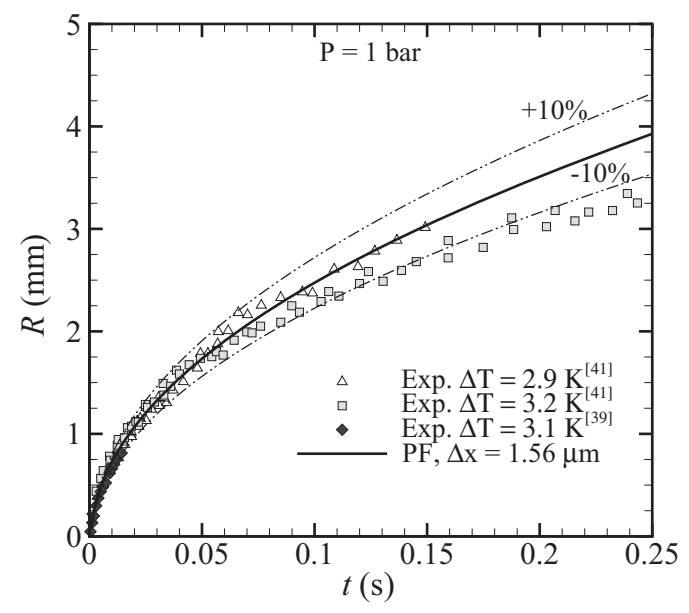

FIG. 15. Comparison between phase-field simulations and experimental results $[39,41]$ for water at atmospheric pressure. Experiments are diplayed in symbols and phase-field simulations are shown with continuous lines.

data agrees well with the uncertainty estimations made by Dergarabedian. To obtain the initial temperature distribution, only the experimental data from Dergarabedian corresponding to a superheating of $\Delta T_{\infty}=3.1 \mathrm{~K}$, were considered. The initial bubble size was $R\left(t_{i}\right)=0.1 \mathrm{~mm}$, the system size was $4.5 \mathrm{~mm}$, and the grid spacing was set to $\Delta_{x}=1.576 \mu \mathrm{m}$.

The last validation case considered ethanol at $P=1$ bar and $\Delta T_{\infty}=3.3 \mathrm{~K}$. The initial bubble size was set to $R\left(t_{i}\right)=$ $0.29 \mathrm{~mm}$, the system size is $3.3 \mathrm{~mm}$, and independence from the grid size was achieved for $\Delta x=16.5 \mu \mathrm{m}$. The predicted interface position and experimental results are presented in Fig. 16. Despite that the bubble grows almost eightfold, the number of grids points required to converge the results is not as high as that in the previous experiment. The main reason for this is the lower interface velocity and the smaller ratio between the bubble size and the thermal boundary layer thickness $R / \delta_{T}=12.4$.

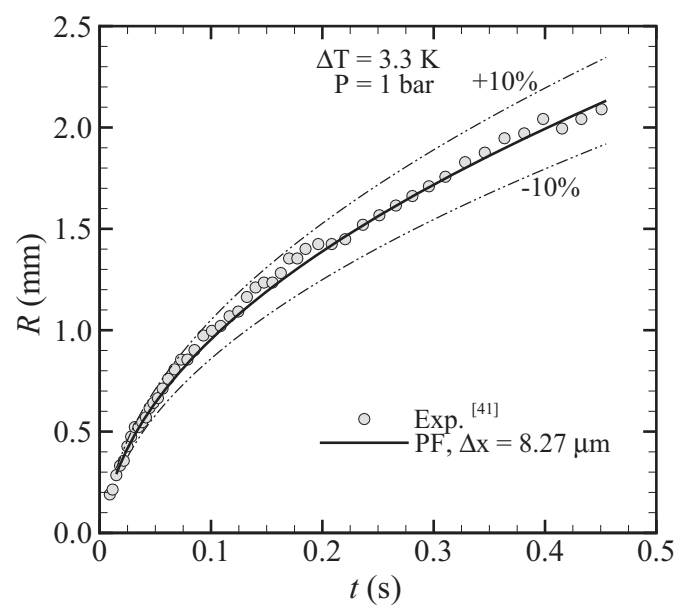

FIG. 16. Comparison between phase-field simulations and experimental results [41] for ethanol at atmospheric pressure. Experiments are displayed in symbols and phase-field simulations are shown with continuous lines. 


\section{CONCLUSIONS}

A new quantitative phase-field model has been developed to study liquid-vapor phase transitions. The model accounts for surface tension effects and large property contrast. A phenomenological antitrapping current, equivalent to that derived originally by Karma [13] and later by Ohno and Matsuura [16] for dendritic growth controlled by a solutal boundary layer, was introduced to eliminate spurious effects in the model, such us interface stretching and surface diffusion. Numerical simulations (not presented in the paper) have shown that the use of this current increases the convergence rate towards the sharp-interface limit. The asymptotic analysis of the current model revealed a very small thermal capillary length, which makes simulations in the millimeter range not feasible. Conventional models derived for solidification require an interface thickness of the same order of magnitude as the solutal capillary length to accurately account for the changes in the interface temperature and concentration due to curvature effects (Gibbs-Thomson relation). Since in solidification the surface energy is anisotropic, an accurate modeling and simulation of curvature-related phenomena is crucial to capture quantitatively the evolution of the interface during dendritic growth. In contrast, during bubble growth in infinite mediums, the surface energy is isotropic and the curvature effects, on the interface temperature, are negligible for bubble sizes of practical interest. Based on this fact, the curvature was eliminated from the potential controlling the phase change, but it was kept in the potential accounting for surface tension. These two potentials are required to handle capillary effects and phase change independently. The elimination of the curvature leads to a change in the length scale dominating the phase change from the capillary length to the thermal boundary layer thickness, which is several orders of magnitude larger. This permits quantitative simulations of bubble growth in the millimeter range.

Comparison of phase-field simulations with analytical solutions, for various one-dimensional problems, are in very good agreement in terms of interface position and temperature distribution. The convergence study, based on the relative error on the growth exponent, showed that the model converges quadratically towards the sharp-interface limit for very fine grids, as predicted from the asymptotic analysis. Nonetheless, for coarser grids, the error increases at a lower rate than the quadratic behavior displayed by fine grids, which, in turn, leads to accurate predictions of the interface position, even for coarse grids. To further validate the model, a comparison with experimental data for single bubble growth in the diffusion-controlled regime was carried out. The results revealed an excellent agreement between predicted and experimentally measured bubble size for water and ethanol, under different levels of superheating and system pressures. The good results found in the comparison between phase-field simulations with analytical solutions and experimental results support the validity of the model.

The present model constitutes the first step towards the full description of single or multiple bubbles, growing from a hot surface, under convective conditions. A new phase-field model is currently being developed to deal with evaporation phenomena at the contact line. Fully three-dimensional simulations of bubble growth in a superheated liquid are currently being carried out and the results will be presented in a forthcoming publication.

\section{ACKNOWLEDGMENTS}

The author thanks Dr. Yohei Sato for valuable discussions on interface tracking techniques. This work was partially funded by Swissnuclear, through the project "Multi-Scale Modeling Analysis of convective boiling (MSMA)."

\section{APPENDIX A: THE UNDERLYING FUNDAMENTAL IDEA BEHIND PHASE-FIELD MODELS}

The fundamental idea behind all phase-field models is simple, elegant, and universal, regardless of the phase change under study, and it could be synthesized as "All the processes in nature occur in order to minimize the free energy of the system." Certainly, someone could refute this statement by simply referring to the case where an electron in a single atom could jump from a lower to a higher energy level, due to quantum mechanical effects. Nonetheless, in average, the electron will spend much more time at the lower energy level. Therefore by "processes in nature," I am referring specifically to the average behavior of nature in a measurable time scale. Thermodynamics provides us with a powerful tool to calculate the free energy of a system and, by comparing two different states, we can identify which of them is more stable, but the path to go from a higher to the lower energy state is out of the realm of thermodynamics. This brings up the dilemma of how to introduce time in our understanding of nature. If, for instance, the free energy of a system subjected to several constrains is denoted by $\mathcal{H}$, its time derivative must be negative if the system evolves from a high energy level to a lower one (Lyapunov-type functional). This is mathematically stated as

$$
\frac{d \mathcal{H}}{d t}<0 \text {. }
$$

If $\mathcal{H}$ can only change by changing the distribution of an order parameter $\phi$, this inequality in general terms is expressed as

$$
\int_{\Omega}\left(\frac{\delta \mathcal{H}}{\delta \phi} \frac{\partial \phi}{\partial t}\right) d \Omega<0
$$

where $\delta$ is the functional derivative and $\Omega$ is the system volume. For a nonconserved order parameter, this inequality can be satisfied if the evolution of the order parameter is given by

$$
\frac{\partial \phi}{\partial t}=-\sum_{i} M_{i}\left(\frac{\delta \mathcal{H}}{\delta \phi}\right)^{i}
$$

where $i=1,3,5, \ldots$ and $M_{i}>0$. For systems close to equilibrium, the driving force for the transformation $\delta \mathcal{H} / \delta \phi$ is small and, thus, the linear term will dominate the evolution of the order parameter. On the contrary, if $\delta \mathcal{H} / \delta \phi$ is large, higher-order terms become important and might bring the system into a metastable state or drive the transformation by a different means other than through the evolution of an interface. The Allen-Cahn equation is obtained by neglecting higher-order terms. The temporal evolution of a conserved order parameter must satisfy a continuity equation, thus, $\partial_{t} \phi=-\nabla \cdot \mathbf{J}_{\phi}$, where $\mathbf{J}_{\phi}$ is the total net flux of $\phi$. Substituting 
this continuity equation in Eq. (A2) yields

$$
-\int_{\Omega}\left(\frac{\delta \mathcal{H}}{\delta \phi} \nabla \cdot \mathbf{J}_{\phi}\right) d \Omega<0
$$

By applying the divergence theorem and impeding the exchange of $\phi$ with the surrounding $\left(\mathbf{J}_{\phi}=0\right.$ at the boundaries), the last inequality can be written as

$$
\int_{\Omega}\left(\mathbf{J}_{\phi} \cdot \nabla\left(\frac{\delta \mathcal{H}}{\delta \phi}\right)\right) d \Omega<0,
$$

which is satisfied if we define the total flux as

$$
\mathbf{J}_{\phi}=-\sum_{i} M_{i}\left(\nabla\left(\frac{\delta \mathcal{H}}{\delta \phi}\right)\right)^{i} \nabla\left(\frac{\delta \mathcal{H}}{\delta \phi}\right),
$$

where $i=0,2,4, \ldots$ and $M_{i}>0$. The well-known CahnHilliard equation is obtained by replacing the total net flux into the continuity equation for the order parameter and neglecting higher-order terms. This derivations illustrate that by using "intuitive" arguments such as the one involved in Eq. (A2), we can derive two fundamental equations with a wide range of applications. All phase-field models rely on these two equations to study the evolution of one or a set of (conserved and nonconserved) order parameters that decrease the free energy of the system. Thus, the main difference among the models does not fall on the derivation of the phase-field equation itself but in the details of the free-energy functional and the subtleties in the coupling between the phase field and the rest of the field equations entering the model (continuity equation for mass, energy, linear momentum, etc.). In solidification as well as boiling, the order parameter is nonconserved and, therefore, the evolution of the phase field is governed by the Allen-Cahn equation. Nevertheless, there are small differences that change significantly the outcome of the models. For instance, the anisotropy of the surface energy is a key property in solidification and, without it, dendritic growth would not be possible. Consequently, a considerable amount of effort must be put on modeling correctly the surface energy anisotropy. In the case of boiling, bubbles growing away from a wall do not experience a surface energy anisotropy, and, therefore, the relevant phenomenon to be modeled is surface tension force, which is fundamental to predict correctly bubble deformation under a pressure gradient. The situation might differ completely when bubbles are attached to a hot surface, where atomic interactions between the wall and fluid molecules should induce a lost of symmetry of the surface energy in the proximity of the contact line, which, at the same time, could translate into an abrupt change of the liquid-vapor interface curvature, without a change in the capillary pressure.

\section{APPENDIX B: THREE-DIMENSIONAL ORTHOGONAL COORDINATES}

For the asymptotic analysis of the phase-field model, a suitable coordinated system is required. Two-dimensional systems have already been used for this purpose, but, to the best of my knowledge, no such systems have been extended to three dimensions. In the orthogonal system presented in this work, any variation of a point $\mathbf{x}$ can be expressed as a displacement of the point $\mathbf{x}_{0}$ along the surface plus a variation

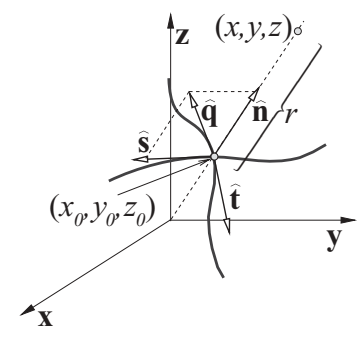

FIG. 17. Orthogonal coordinate system

in the normal direction, thus,

$$
\mathbf{x}=\mathbf{x}_{0}+r \hat{\mathbf{n}} .
$$

The system is composed of a normal $\hat{\mathbf{n}}$ and two tangential directions $\hat{\mathbf{s}}$ and $\hat{\mathbf{t}}$. If the net heat flux to the interface $\mathbf{q}$ is parallel to the normal vector, the tangential directions can be selected arbitrarily. On the contrary, if the heat flux is not parallel to the normal vector, the $\hat{\mathbf{s}}$ direction has been chosen such as that it points in the same direction of the projected heat flux on the surface, which eliminates the heat flux component in the $\hat{\mathbf{t}}$ direction. Using Einstein's notation, the metric tensor for any orthogonal coordinate system is obtained from $g_{i k}=j_{m i} j_{m k}$, where $g_{i k}$ are the components of the metric tensor and $j_{m k}$ the components of the Jacobian matrix. It can be proved that for the current orthogonal system, the metric tensor is

$$
\stackrel{\leftrightarrow}{\mathbf{g}}=\left(\begin{array}{ccc}
1 & 0 & 0 \\
0 & \left(1+r \kappa_{s}\right)^{2} & 0 \\
0 & 0 & \left(1+r \kappa_{t}\right)^{2}
\end{array}\right)
$$

where $\kappa_{s}$ and $\kappa_{t}$ are the curvatures along the tangential directions $s$ and $t$ (see Fig. 17). Thus, the Lamé coefficients are $h_{n}=1, h_{s}=\left|1+r \kappa_{s}\right|$, and $h_{t}=\left|1+r \kappa_{t}\right|$. With this, the gradient and divergence operator are defined as

$$
\begin{gathered}
\nabla \varphi=\frac{1}{h_{n}} \frac{\partial \varphi}{\partial r} \hat{\mathbf{n}}+\frac{1}{h_{s}} \frac{\partial \varphi}{\partial s} \hat{\mathbf{s}}+\frac{1}{h_{t}} \frac{\partial \varphi}{\partial t} \hat{\mathbf{t}}, \\
\nabla \cdot \mathbf{f}=\frac{1}{h_{n} h_{s} h_{t}}\left\{\frac{\partial}{\partial r}\left(h_{s} h_{t} f_{n}\right)+\frac{\partial}{\partial s}\left(h_{r} h_{t} f_{s}\right)+\frac{\partial}{\partial t}\left(h_{n} h_{s} f_{t}\right)\right\} .
\end{gathered}
$$

Since the component of the heat flux in the $\mathbf{t}$ direction is zero, the divergence of the heat flux reads

$$
\begin{aligned}
\nabla \cdot\left(k(\phi) \nabla T+\mathbf{j}_{n}\right)= & \frac{\partial}{\partial r}\left(k(\phi) \frac{\partial T}{\partial r}+j_{n}\right)+k(\phi) \frac{\partial T}{\partial r} \frac{1}{h_{s} h_{t}} \\
& \times \frac{\partial}{\partial r}\left(h_{s} h_{r}\right)+\frac{1}{h_{s}^{2}} \frac{\partial}{\partial s}\left(k(\phi) \frac{\partial T}{\partial s}\right),
\end{aligned}
$$

with

$$
\frac{1}{h_{s} h_{t}} \frac{\partial\left(h_{s} h_{t}\right)}{\partial r}=\left(\kappa_{s}+\kappa_{t}\right)-r\left(\kappa_{s}^{2}+\kappa_{t}^{2}\right)+O\left(\left(r \kappa_{s}\right)^{2}+\left(r \kappa_{t}\right)^{2}\right)
$$

and

$$
\frac{1}{h_{s}^{2}}=\frac{1}{\left(1+r \kappa_{s}\right)^{2}}=1-2 r \kappa_{s}+O\left(\left(r \kappa_{s}\right)^{2}\right)
$$


Dropping higher-order terms and making use of the total $\kappa=\kappa_{s}+\kappa_{t}$ and Gaussian $\kappa_{g}$ curvatures, Eq. (B6) can be approximated by

$$
\frac{1}{h_{s} h_{t}} \frac{\partial\left(h_{s} h_{t}\right)}{\partial r} \approx \kappa\left(1-\frac{\kappa}{2} r\right) .
$$

Using an arbitrary length scale $l_{c}$, the dimensionless form of the divergence of the heat flux reads

$$
\begin{aligned}
\breve{\nabla} \cdot\left(k(\phi) \breve{\nabla} T+\breve{\mathbf{j}}_{n}\right)= & \frac{\partial}{\partial \breve{r}}\left(k(\phi) \frac{\partial T}{\partial \breve{r}}+\breve{j}_{n}\right) \\
& +\left(\breve{j}_{n}+k(\phi) \frac{\partial T}{\partial \breve{r}} \breve{\kappa}\right)\left(1-\frac{\breve{\kappa}}{2} \breve{r}\right) \\
& +\frac{\partial}{\partial \breve{s}}\left(k(\phi) \frac{\partial T}{\partial \breve{s}}\right)\left(1-2 \breve{r} \breve{\kappa}_{s}\right),
\end{aligned}
$$

where $\breve{\kappa}=\kappa l_{c}$ and $\breve{\kappa}_{s}=\kappa_{s} l_{c}$ are dimensionless curvatures. In terms of the stretched variable $\eta=r / W=\breve{r} / \epsilon$, Eq. (B9) reads

$$
\begin{aligned}
\tilde{\nabla} \cdot( & \left.k(\phi) \tilde{\nabla} T+\tilde{\mathbf{j}}_{n}\right) \\
= & \frac{\partial}{\partial \eta}\left(k(\phi) \frac{\partial T}{\partial \eta}\right)+\epsilon\left(\breve{\kappa} k(\phi) \frac{\partial T}{\partial \eta}+\frac{\partial \breve{j}_{n}}{\partial \eta}\right) \\
& +\epsilon^{2}\left(\frac{\partial}{\partial \breve{s}}\left(k(\phi) \frac{\partial T}{\partial \breve{s}}\right)-k(\phi) \frac{\partial T}{\partial \eta} \frac{\breve{\kappa}^{2}}{2} \eta+\breve{j}_{n} \breve{\kappa}\right)+O\left(\epsilon^{3}\right) .
\end{aligned}
$$

The Laplacian of the phase field is given by

$$
\tilde{\nabla}^{2} \psi=\frac{\partial^{2} \psi}{\partial \eta^{2}}+\epsilon \breve{\kappa} \frac{\partial \psi}{\partial \eta}-\frac{\epsilon^{2} \breve{\kappa}^{2}}{2} \eta \frac{\partial \psi}{\partial \eta}+O\left(\epsilon^{3}\right) .
$$

\section{APPENDIX C: RELEVANT INTEGRALS}

All the integrals presented in this appendix are based on the zeroth-order phase field, given by the hyperbolic tangent $\psi_{0}=\tanh (\eta / \sqrt{2})$. In addition, the limits for the function $p^{(0)}$ are $p_{+}^{(0)}=(1-\Phi) k_{v} / k_{l}$ and $p_{-}^{(0)}=-\Phi$

$$
\int_{0}^{\eta} p^{(0)} d \eta=p_{+}^{(0)} \eta-\left(\frac{p_{+}^{(0)}-p_{-}^{(0)}}{2}\right) \sqrt{2} \ln \left(\psi_{0}+1\right),
$$

$$
\begin{gathered}
I_{0}=\frac{2 \sqrt{2}}{3} \\
K_{0}=-\left(p_{+}^{(0)}-p_{-}^{(0)}\right)\left(\frac{2 \ln 2}{3}-\frac{5}{9}\right), \\
F^{ \pm}=F=\left(\frac{p_{+}^{(0)}-p_{-}^{(0)}}{2}\right) \sqrt{2} \ln 2 .
\end{gathered}
$$

[1] C. Wright, J. O’Brien, and R. Spall, Int. J. Heat Mass Transf. 51, 1071 (2008).

[2] L. Cheng and J. Thome, Appl. Therm. Eng. 29, 2426 (2009).

[3] T. Fuchs, J. Kern, and P. Stephan, J. Heat Transf. 128, 1257 (2006).

[4] D. Li and V. Dhir, J. Heat Transf. 129, 864 (2007).

[5] J. Myers, V. Yerramilli, S. Hussey, G. Yee, and J. Kim, Int. J. Heat Mass Transf. 48, 2429 (2007).

[6] L. Rayleigh, Philos. Mag. 34, 94 (1917).

[7] S. Welch and J. Wilson, J. Comput. Phys. 160, 662 (2000).

[8] Y. Sato and B. Ničeno, Int. J. Numer. Meth. Fluids 70, 441 (2012).

[9] F. Gibou, L. Chen, D. Nguyen, and S. Banerjee, J. Comput. Phys. 222, 536 (2007).

[10] D. Juric and G. Tryggvason, Int. J. Multiphas. Flow 24, 387 (1998).

[11] T. Ye, W. Shyy, and J. Chung, J. Comput. Phys. 174, 781 (1998).

[12] K. Tsujimoto, A. Nakamura, T. Shakouchi, and T. Ando, in Proceedings of the 7th International Conference on Multiphase Flow (ICMF, University of Florida, Florida, 2010).

[13] A. Karma, Phys. Rev. Lett. 87, P115701 (2001).

[14] C. Beckermann, H. Diepers, I. Steinbach, A. Karma, and X. Tong, J. Comput. Phys. 154, 468 (1999).

[15] J. C. Ramirez, C. Beckermann, A. Karma, and H. J. Diepers, Phys. Rev. E 69, 051607 (2004).

[16] M. Ohno and K. Matsuura, Phys. Rev. E 79, 031603 (2009).

[17] N. Zhou, C. Shen, M. Mills, and Y. Wang, Acta Mater. 55, 5369 (2007).

[18] A. Kazaryan, Y. Wang, S. Dregia, and B. Patton, Acta Mater. 50, 2491 (2002).
[19] S. Wise, J. Lowengrub, J. Kima, and W. Johnson, Superlattice. Microst. 36, 293 (2004)

[20] V. Badalassi, H. Ceniceros, and S. Banerjee, J. Comput. Phys. 190, 371 (2003).

[21] Q. He and N. Kasagi, Fluid Dyn. Res. 40, 497 (2008).

[22] R. Borcia and M. Bestehorn, Phys. Rev. E 75, 056309 (2007).

[23] Y. Sun and C. Beckermann, J. Comput. Phys. 220, 626 (2007).

[24] R. Borcia and M. Bestehorn, Eur. Phys. J. B 44, 101 (2005).

[25] D. Jamet, O. Lebaigue, N. Coutris, and J. Delhaye, J. Comput. Phys. 169, 624 (2001).

[26] A. Karma and W. J. Rappel, Phys. Rev. E 57, 4323 (1998).

[27] Y. Sun and C. Beckermann, Physica D 198, 281 (2004).

[28] B. Shi, S. Sinha, and V. Dhir, J. Chem. Phys. 124, 204715 (2006).

[29] J. Ni and C. Beckermann, Metall. Trans. B 22, 349 (1991).

[30] S. Hardt and F. Wondra, J. Comput. Phys. 227, 5871 (2008).

[31] J. Kirkwood and F. Buff, J. Chem. Phys. 17, 338 (1949).

[32] A. Yang, P. Fleming, and J. Gibbs, J. Chem. Phys. 64, 3732 (1976).

[33] J. Cahn and J. Hilliard, J. Chem. Phys. 28, 258 (1958).

[34] A. Badillo and C. Beckermann, Acta Mater. 54, 2015 (2006).

[35] E. Olsson and G. Kreiss, J. Comput. Phys. 210, 225 (2005).

[36] G. McFadden, A. Wheeler, and D. Anderson, Phyica D 144, 154 (2000).

[37] L. Scriven, Chem. Eng. Sci. 10, 1 (1959).

[38] M. Plesset and S. Zwick, J. Appl. Phys. 23, 95 (1952).

[39] P. Dergarabedian, Ph.D. thesis, Caltech, 1952.

[40] Y. Lien, Ph.D. thesis, M.I.T., 1969.

[41] L. Florschuetz, C. Henry, and A. R. Khan, Int. J. Heat Mass Transf. 12, 1465 (1969). 